\title{
Computer-assisted automated synthesis. III. Synthesis of substituted N-(carboxyalkyl) amino-acid tert-butyl ester derivatives
}

\author{
Nobuyoshi Hayashi*, Tohru Sugawara and Shinji \\ Kato \\ Chemistry Research Laboratories, Research and Development \\ Division, Takeda Chemical Industries Ltd, Juso Honmachi 2- \\ chome, Yodogawaku, Osaka 532, Japan
}

$A$ versatile automated synthesis apparatus, equipped with a chemical artificial intelligence, was developed to prepare and isolate a wide variety of compounds. The apparatus was to the synthesis of substituted $N$-(carboxyalkyl)amino-acids. The apparatus [1,2] is composed of units for performing various tasks, for example reagent supply, reaction, purification and separation, each linked to a control system. All synthetic processes, including washing and drying of the apparatus after each synthetic run, were automatically performed from the mixing of the reactants to the isolation of the products as powders or crystals. The reaction of an amino-acid tertbutyl ester acetic acid salt with a 2-keto acid sodium salt produces an unstable intermediate, Schiff base, which is reduced with sodum cyanoborohydride to give a substituted $N$-(carboxyalkyl)aminoacid tert-butyl ester sodium salt. The equilibrium and the consecutive reactions were controlled by adding sodium cyanoborohydride using the artificial intelligence software, which contained novel kinetic equations [3] and substituent effects [4].

Substitued $N$-(carboxyalkyl)amino-acid tert-butyl esters, 90 derivatives, were automatically synthesized using the computerassisted automated synthesis apparatus. The syntheses were performed unattended 24 hours a day, except for supplying the raw materials, reagents and solvents. The apparatus is extremely valuable for synthesizing many derivatives of a particular compound. The configurations of the products were determined by circular dichroism measurements.

\section{Introduction}

In general, organic syntheses are time-consuming; involving the preliminary estimation of the reaction conditions, mixing of the raw materials and reagents, control of the reaction conditions, concentration, extraction, purification and finally isolation of the desired products. Although some research on laboratory automation, especially for synthesis, has been attempted [5-8], an automatic apparatus for the estimation of the optimum reaction conditions, as well as control of all the synthetic processes, has remained an important objective. The authors recently reported a reaction-control methods based on the novel kinetic equations and substituent effect, which made up the computer software for the automated apparatus, and the design and construction of fully automated synthesis apparatus [1].

This paper deals with an application of the apparatus for the synthesis of substituted $\mathrm{N}$-(carboxyalkyl)aminoacids - known as 'unusual amino acids' [9]. Several of these unusual amino-acids have been found to occur in nature; for example strombine, an active fish attractant extracted from Strombus gigas [10], and N-(carboxymethyl)-L-serine which has been isolated from asparagus shoots [11]. However, the only derivatives of N-(carboxyalkyl)amoni-acids which have been synthesized, are a series of $\mathrm{N}$-(carboxymethyl)amino-acids [12].

The reaction of amino-acid tert-butyl ester acetic acid salt (1) with keto acid (2) in methanol gives an unstable intermediate. Schiff base, which is then reduced with sodium cyanoborohydride to afford the N(carboxyalkyl)amino-acid tert-butyl ester (3). The products obtained from natural L-amino acids, all of which have an S-configuration on the chiral centre, except for cysteine and methionine, were generally a mixture of diastereoisomers $[(\mathrm{S}, \mathrm{R})$ - and $(\mathrm{S}, \mathrm{S})$-isomers $]$. As the diastereoisomers were separated, their configurations were also determined by means of circular dichroism (CD) mesurement.

\section{Results and discussion}

\section{Procedure for the automated synthesis}

The automated synthesis apparatus used is shown in figure 1. All synthetic processes, including washing and drying of the apparatus after a synthetic run, were automatically performed; from the mixing of reactants to<smiles>[R][R](=O)C([R])O[R](=O)O[Na]</smiles>

(1)<smiles>[R16]OC(=O)C([R])NC([R])[N+]([O-])([O-])OCc1ccccc1</smiles>

(3)

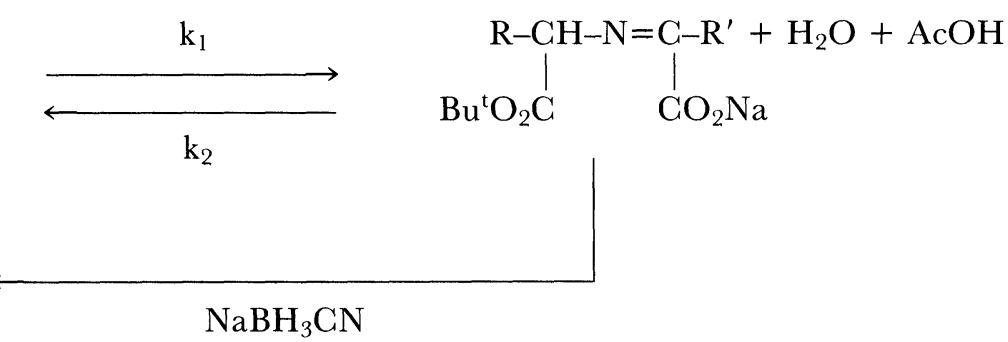

$\mathrm{NaBH}_{3} \mathrm{CN}$ 


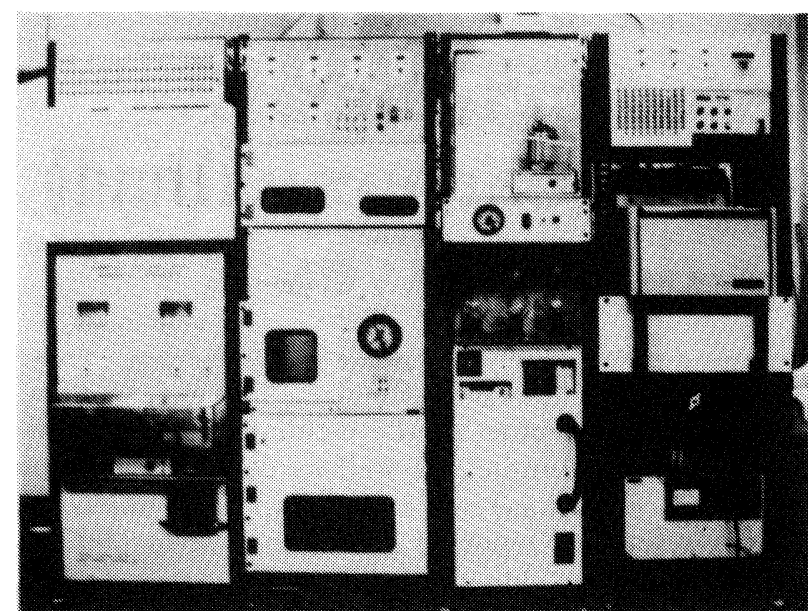

Figure 1. The automated synthesis apparatus.

the isolation of the products as powders. The prediction of the optimum reaction conditions and the reaction control in real time, are accomplished using the novel kinetic equations and substituent effect which make up the computer software, as previously reported [4].

As a typical automated synthesis using the apparatus, the reaction of L-leucine tert-butyl ester acetic acid salt (4) with 2-ketoisocaproic acid sodium salt (5) in methanol was accomplished in the following manner. Methanolic solutions of $4(0.8 \mathrm{~mol} / \mathrm{l}), 5(0.4 \mathrm{~mol} / \mathrm{l})$, sodium cyanoborohydride $(0.2 \mathrm{~mol} / \mathrm{l})$ and sodium hydroxide $(0.8 \mathrm{~mol} /$ 1; $\mathrm{MeOH}: \mathrm{H}_{2} \mathrm{O}=1: 1, \mathrm{v} / \mathrm{v}$ ) were stored in the designated reservoirs. A flowchart showing the sequence of operations performed for a synthesis of operations performed for a synthesis is presented in figure 2. Under computer control, $5 \mathrm{ml}$ of 4 and $10 \mathrm{ml}$ of 5 were volumetrically measured and added to the reaction flask. The addition rate of sodium cyanoborohydride was controlled as shown in table 1 .

The computer software directed the addition of sodium cyanoborohydride until the rate of decrease of the chemical yield against the reaction time reached $0 \cdot 1 \% /$ min, on the basis of the kinetic equations. The reaction was then terminated $10 \mathrm{~min}$ after the final addition. Thus

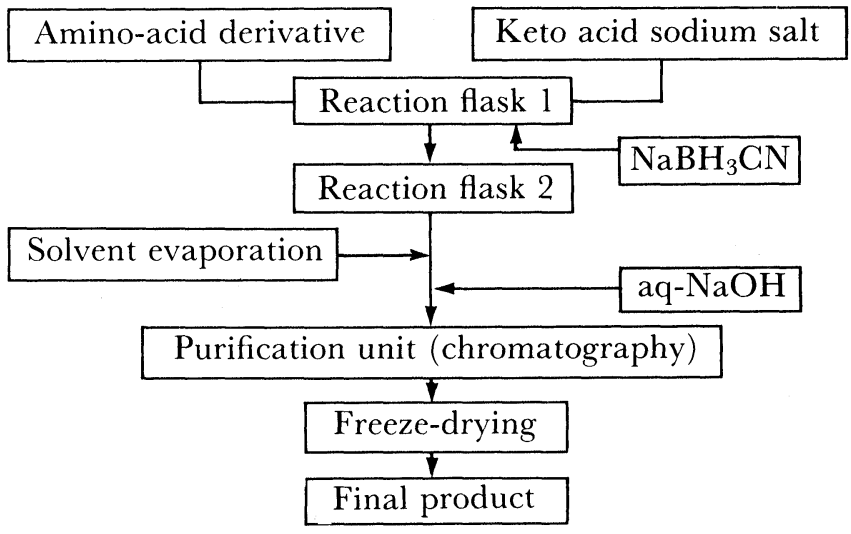

Figure 2. The operation sequence of the automation system.

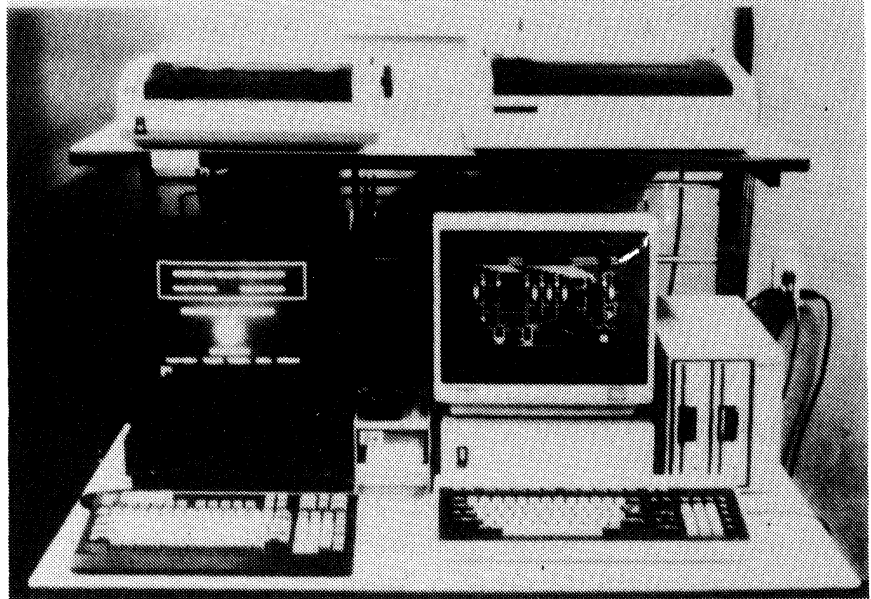

Table 1. The addition rate of sodium cyanoborohydride.

\begin{tabular}{cc}
\hline Time (min) & $\begin{array}{c}\text { Addition } \\
\text { volume }(\mathrm{ml})\end{array}$ \\
\hline $4 \cdot 7$ & $4 \cdot 3$ \\
$13 \cdot 6$ & $2 \cdot 9$ \\
$28 \cdot 5$ & $2 \cdot 0$ \\
$52 \cdot 4$ & $1 \cdot 4$ \\
$89 \cdot 3$ & $0 \cdot 9$ \\
$145 \cdot 2$ & $0 \cdot 6$ \\
$155 \cdot 2$ & Finish \\
\hline
\end{tabular}

the reaction was stopped after $155 \mathrm{~min}$, and the resulting mixture was transferred to the concentration flask. After evaporation to dryness under reduced pressure, $10 \mathrm{ml}$ of aqueous sodium hydroxide was added to the residue with stirring. The solution was then injected into the purification unit. The high performance liquid chromatographic (HPLC) chart, which was displayed on a CRT monitor during the purification stage, is presented in figure 3 .

The peak at the retention time $(27.2 \mathrm{~min})$ was not clearly identified but it was attributed to decomposition products of the sodium cyanoborohydride and the keto acid. The two peaks having retention times of 52.3 and $66.3 \mathrm{~min}$ were identical to those of the diastereoisomers of N-(3methyl-1-sodioxycarbonyl)butyl-L-leucince tert-butyl ester (6). The analytical yield calculated from the area of these two peaks was almost quantitative on the basis of

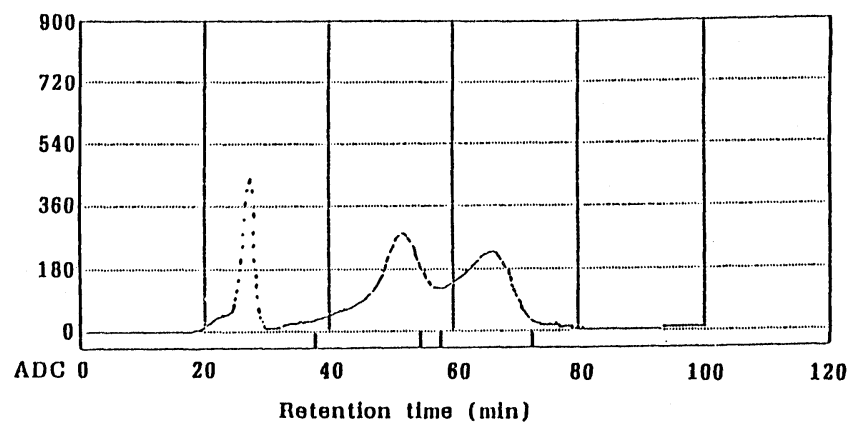

Figure 3. HPLC chart of the reaction mixture. 
Table 2. Chemical yields and the elemental analysis.

\begin{tabular}{|c|c|c|c|c|c|c|c|c|c|c|}
\hline \multirow{3}{*}{$\frac{\mathrm{R}_{1}}{\mathrm{Et}}$} & \multirow{3}{*}{$\begin{array}{c}\mathrm{R}_{2} \\
\mathrm{H}\end{array}$} & \multirow{3}{*}{$\begin{array}{c}\begin{array}{c}\text { Yield } \\
(\%)\end{array} \\
64 \cdot 5\end{array}$} & \multirow{2}{*}{\multicolumn{2}{|c|}{ Formula }} & \multicolumn{3}{|c|}{ Calcd. (\%) } & \multicolumn{3}{|c|}{ Found (\%) } \\
\hline & & & & & \multirow{2}{*}{$\frac{\mathrm{C}}{45 \cdot 11}$} & \multirow{2}{*}{$\frac{\mathrm{H}}{7.95}$} & \multirow{2}{*}{$\frac{\mathrm{N}}{5 \cdot 26}$} & \multirow{2}{*}{$\frac{C}{44.94}$} & \multirow{2}{*}{$\frac{H}{7 \cdot 66}$} & \multirow{2}{*}{$\frac{N}{5 \cdot 55}$} \\
\hline & & & $\mathrm{C}_{10} \mathrm{H}_{18} \mathrm{NNaO}_{4}$ & $1.5 \mathrm{H}_{2} \mathrm{O}$ & & & & & & \\
\hline & $\mathrm{Me}(\mathrm{M})$ & $65 \cdot 2$ & $\mathrm{C}_{11} \mathrm{H}_{20} \mathrm{NNaO}_{4}$ & $2 \cdot 0 \mathrm{H}_{2} \mathrm{O}$ & $45 \cdot 67$ & $8 \cdot 36$ & $4 \cdot 84$ & $45 \cdot 36$ & $7 \cdot 98$ & $4 \cdot 84$ \\
\hline & Et $(\mathrm{A})$ & $76 \cdot 2$ & $\mathrm{C}_{12} \mathrm{H}_{22} \mathrm{NNaO}_{4}$ & $0.4 \mathrm{H}_{2} \mathrm{O}$ & $52 \cdot 51$ & $8 \cdot 37$ & $5 \cdot 10$ & $52 \cdot 68$ & $8 \cdot 13$ & $4 \cdot 96$ \\
\hline & (B) & & $\mathrm{C}_{12} \mathrm{H}_{22} \mathrm{NNaO}_{4}$ & $0.7 \mathrm{H}_{2} \mathrm{O}$ & $51 \cdot 49$ & $8 \cdot 43$ & $5 \cdot 00$ & $51 \cdot 64$ & $8 \cdot 41$ & $5 \cdot 05$ \\
\hline & $\mathrm{n}-\operatorname{Pr}(\mathrm{A})$ & $73 \cdot 9$ & $\mathrm{C}_{13} \mathrm{H}_{24} \mathrm{NNaO}_{4}$ & $0.3 \mathrm{H}_{2} \mathrm{O}$ & $54 \cdot 46$ & $8 \cdot 65$ & $4 \cdot 88$ & $54 \cdot 76$ & $8 \cdot 53$ & $4 \cdot 86$ \\
\hline & (B) & & $\mathrm{C}_{13} \mathrm{H}_{24} \mathrm{NNaO}_{4}$ & $0.4 \mathrm{H}_{2} \mathrm{O}$ & $54 \cdot 12$ & $8 \cdot 66$ & $4 \cdot 85$ & $54 \cdot 17$ & $8 \cdot 69$ & $4 \cdot 78$ \\
\hline & $\mathrm{i}-\operatorname{Pr}(\mathrm{A})$ & $59 \cdot 5$ & $\mathrm{C}_{13} \mathrm{H}_{24} \mathrm{NNaO}_{4}$ & $0.4 \mathrm{H}_{2} \mathrm{O}$ & $54 \cdot 12$ & $8 \cdot 66$ & $4 \cdot 85$ & $54 \cdot 18$ & $8 \cdot 74$ & 4.99 \\
\hline & (B) & & $\mathrm{C}_{13} \mathrm{H}_{24} \mathrm{NNaO}_{4}$ & $0 \cdot 2 \mathrm{H}_{2} \mathrm{O}$ & $54 \cdot 80$ & $8 \cdot 63$ & $4 \cdot 92$ & $54 \cdot 93$ & $8 \cdot 69$ & $4 \cdot 98$ \\
\hline & $\mathrm{n}-\mathrm{Bu}(\mathrm{A})$ & $78 \cdot 8$ & $\mathrm{C}_{14} \mathrm{H}_{26} \mathrm{NNaO}_{4}$ & & 56.93 & $8 \cdot 89$ & $4 \cdot 73$ & $56 \cdot 70$ & $8 \cdot 67$ & $4 \cdot 79$ \\
\hline & (B) & & $\mathrm{C}_{14} \mathrm{H}_{26} \mathrm{NNaO}_{4}$ & & $56 \cdot 93$ & $8 \cdot 89$ & $4 \cdot 74$ & $56 \cdot 83$ & $9 \cdot 13$ & $4 \cdot 79$ \\
\hline & $\mathrm{i}-\mathrm{Bu}(\mathrm{A})$ & $78 \cdot 5$ & $\mathrm{C}_{14} \mathrm{H}_{26} \mathrm{NNaO}_{4}$ & $0 \cdot 2 \mathrm{H}_{2} \mathrm{O}$ & $56 \cdot 25$ & $8 \cdot 90$ & $4 \cdot 96$ & $56 \cdot 43$ & $9 \cdot 01$ & $4 \cdot 88$ \\
\hline & (B) & & $\mathrm{C}_{14} \mathrm{H}_{26} \mathrm{NNaO}_{4}$ & $0 \cdot 2 \mathrm{H}_{2} \mathrm{O}$ & $56 \cdot 25$ & $8 \cdot 90$ & 4.96 & $56 \cdot 26$ & $8 \cdot 94$ & $4 \cdot 63$ \\
\hline & sec-Bu (A) & $29 \cdot 4$ & $\mathrm{C}_{14} \mathrm{H}_{26} \mathrm{NNaO}_{4}$ & & $56 \cdot 93$ & $8 \cdot 87$ & $4 \cdot 74$ & $57 \cdot 19$ & $9 \cdot 35$ & $4 \cdot 40$ \\
\hline & (B) & & $\mathrm{C}_{14} \mathrm{H}_{26} \mathrm{NNaO}_{4}$ & & $56 \cdot 93$ & $8 \cdot 87$ & $4 \cdot 74$ & $57 \cdot 40$ & $9 \cdot 11$ & $4 \cdot 69$ \\
\hline & $\left(\mathrm{CH}_{2}\right)_{2} \mathrm{CO}_{2} \mathrm{Na}(\mathrm{M})$ & $67 \cdot 1$ & $\mathrm{C}_{13} \mathrm{H}_{21} \mathrm{NNa}_{2} \mathrm{O}_{6}$ & & $46 \cdot 85$ & $6 \cdot 35$ & $4 \cdot 20$ & $45 \cdot 60$ & $6 \cdot 65$ & $4 \cdot 13$ \\
\hline & $\left(\mathrm{CH}_{2}\right)_{3} \mathrm{CO}_{2} \mathrm{Na}(\mathrm{M})$ & $53: 4$ & $\mathrm{C}_{14} \mathrm{H}_{23} \mathrm{NNa}_{2} \mathrm{O}_{6}$ & $1.5 \mathrm{H}_{2} \mathrm{O}$ & $44 \cdot 92$ & $7 \cdot 00$ & $3 \cdot 74$ & $45 \cdot 28$ & $7 \cdot 09$ & $4 \cdot 19$ \\
\hline \multirow[t]{17}{*}{ n-Pr } & $\mathrm{H}$ & $70 \cdot 8$ & $\mathrm{C}_{11} \mathrm{H}_{20} \mathrm{NNaO}_{4}$ & $1 \cdot 0 \mathrm{H}_{2} \mathrm{O}$ & $48 \cdot 70$ & $8 \cdot 17$ & $5 \cdot 16$ & $48 \cdot 20$ & $7 \cdot 80$ & $5 \cdot 31$ \\
\hline & $\mathrm{Me}(\mathbf{M})$ & $55 \cdot 5$ & $\mathrm{C}_{12} \mathrm{H}_{22} \mathrm{NNaO}_{4}$ & $2 \cdot 0 \mathrm{H}_{2} \mathrm{O}$ & $47 \cdot 52$ & $8 \cdot 64$ & $4 \cdot 62$ & $48 \cdot 00$ & $8 \cdot 33$ & $5 \cdot 17$ \\
\hline & Et (A) & $74 \cdot 7$ & $\mathrm{C}_{13} \mathrm{H}_{24} \mathrm{NNaO}_{4}$ & $0 \cdot 3 \mathrm{H}_{2} \mathrm{O}$ & $54 \cdot 46$ & $8 \cdot 65$ & $4 \cdot 88$ & $54 \cdot 77$ & $8 \cdot 90$ & $4 \cdot 90$ \\
\hline & (B) & & $\mathrm{C}_{13} \mathrm{H}_{24} \mathrm{NNaO}_{4}$ & $0.5 \mathrm{H}_{2} \mathrm{O}$ & $53 \cdot 78$ & $8 \cdot 68$ & $4 \cdot 82$ & $53 \cdot 78$ & $8 \cdot 67$ & $4 \cdot 80$ \\
\hline & $\mathrm{n}-\operatorname{Pr}(\mathrm{A})$ & $71 \cdot 5$ & $\mathrm{C}_{14} \mathrm{H}_{26} \mathrm{NNaO}_{4}$ & $0 \cdot 3 \mathrm{H}_{2} \mathrm{O}$ & $55 \cdot 91$ & $8 \cdot 91$ & $4 \cdot 66$ & $56 \cdot 04$ & $9 \cdot 16$ & $4 \cdot 60$ \\
\hline & (B) & & $\mathrm{C}_{14} \mathrm{H}_{26} \mathrm{NNaO}_{4}$ & $0.5 \mathrm{H}_{2} \mathrm{O}$ & $55 \cdot 25$ & $8 \cdot 94$ & $4 \cdot 60$ & $54 \cdot 97$ & $9 \cdot 24$ & $4 \cdot 57$ \\
\hline & i-Pr $(A)$ & $59 \cdot 8$ & $\mathrm{C}_{14} \mathrm{H}_{26} \mathrm{NNaO}_{4}$ & $0.5 \mathrm{H}_{2} \mathrm{O}$ & $55 \cdot 25$ & $8 \cdot 94$ & $4 \cdot 60$ & $55 \cdot 40$ & $8 \cdot 88$ & $4 \cdot 70$ \\
\hline & (B) & & $\mathrm{C}_{14} \mathrm{H}_{26} \mathrm{NNaO}_{4}$ & $0.4 \mathrm{H}_{2} \mathrm{O}$ & $55 \cdot 58$ & $8 \cdot 93$ & $4 \cdot 63$ & $55 \cdot 58$ & $9 \cdot 04$ & $4 \cdot 55$ \\
\hline & $\mathrm{n}-\mathrm{Bu}(\mathrm{A})$ & $75 \cdot 9$ & $\mathrm{C}_{15} \mathrm{H}_{28} \mathrm{NNaO}_{4}$ & $0 \cdot 2 \mathrm{H}_{2} \mathrm{O}$ & $57 \cdot 56$ & $9 \cdot 15$ & $4 \cdot 48$ & $57 \cdot 82$ & $9 \cdot 20$ & $4 \cdot 48$ \\
\hline & (B) & & $\mathrm{C}_{15} \mathrm{H}_{28} \mathrm{NNaO}_{4}$ & $0 \cdot 2 \mathrm{H}_{2} \mathrm{O}$ & $57 \cdot 56$ & $9 \cdot 15$ & $4 \cdot 48$ & $57 \cdot 85$ & 8.91 & $4 \cdot 62$ \\
\hline & $\mathrm{i}-\mathrm{Bu}(\mathrm{A})$ & $77 \cdot 6$ & $\mathrm{C}_{15} \mathrm{H}_{28} \mathrm{NNaO}_{4}$ & $0.4 \mathrm{H}_{2} \mathrm{O}$ & $56 \cdot 91$ & $9 \cdot 17$ & $4 \cdot 42$ & $56 \cdot 94$ & $9 \cdot 03$ & $4 \cdot 50$ \\
\hline & (B) & & $\mathrm{C}_{15} \mathrm{H}_{28} \mathrm{NNaO}_{4}$ & $0 \cdot 4 \mathrm{H}_{2} \mathrm{O}$ & $56 \cdot 91$ & $9 \cdot 17$ & $4 \cdot 42$ & $57 \cdot 03$ & $9 \cdot 22$ & $4 \cdot 60$ \\
\hline & sec-Bu (A) & $37 \cdot 0$ & $\mathrm{C}_{15} \mathrm{H}_{28} \mathrm{NNaO}_{4}$ & $0.8 \mathrm{H}_{2} \mathrm{O}$ & $55 \cdot 82$ & $9 \cdot 24$ & $4 \cdot 34$ & $55 \cdot 51$ & $9 \cdot 01$ & $4 \cdot 86$ \\
\hline & (B) & & $\mathrm{C}_{15} \mathrm{H}_{28} \mathrm{NNaO}_{4}$ & $0.4 \mathrm{H}_{2} \mathrm{O}$ & $56 \cdot 91$ & $9 \cdot 17$ & $4 \cdot 43$ & $56 \cdot 52$ & $8 \cdot 99$ & $4 \cdot 82$ \\
\hline & $\left(\mathrm{CH}_{2}\right)_{2} \mathrm{CO}_{2} \mathrm{Na}(\mathrm{M})$ & $60 \cdot 0$ & $\mathrm{C}_{14} \mathrm{H}_{23} \mathrm{NNa}_{2} \mathrm{O}_{6}$ & $2.0 \mathrm{H}_{2} \mathrm{O}$ & $43 \cdot 86$ & $7 \cdot 09$ & $3 \cdot 65$ & $43 \cdot 42$ & $6 \cdot 86$ & $3 \cdot 57$ \\
\hline & $\left(\mathrm{CH}_{2}\right)_{3} \mathrm{CO}_{2} \mathrm{Na}(\mathrm{A})$ & $55 \cdot 7$ & $\mathrm{C}_{15} \mathrm{H}_{25} \mathrm{NNa}_{2} \mathrm{O}_{6}$ & $2 \cdot 0 \mathrm{H}_{2} \mathrm{O}$ & $45 \cdot 34$ & $7 \cdot 36$ & $3 \cdot 52$ & $45 \cdot 48$ & $7 \cdot 05$ & $3 \cdot 57$ \\
\hline & (B) & & $\mathrm{C}_{15} \mathrm{H}_{25} \mathrm{NNa}_{2} \mathrm{O}_{6}$ & $1.5 \mathrm{H}_{2} \mathrm{O}$ & $46 \cdot 39$ & $7 \cdot 27$ & $3 \cdot 61$ & $46 \cdot 36$ & $7 \cdot 03$ & $3 \cdot 86$ \\
\hline i-Pr & $\mathrm{H}$ & $84 \cdot 1$ & $\mathrm{C}_{11} \mathrm{H}_{20} \mathrm{NNaO}_{4}$ & $0.5 \mathrm{H}_{2} \mathrm{O}$ & $50 \cdot 37$ & $8 \cdot 07$ & $5 \cdot 34$ & $50 \cdot 08$ & $7 \cdot 88$ & $5 \cdot 59$ \\
\hline & $\mathrm{Me}(\mathrm{M})$ & 52.8 & $\mathrm{C}_{12} \mathrm{H}_{22} \mathrm{NNaO}_{4}$ & $1 \cdot 0 \mathrm{H}_{2} \mathrm{O}$ & $50 \cdot 52$ & $8 \cdot 48$ & $4 \cdot 91$ & $50 \cdot 02$ & $8 \cdot 15$ & $5 \cdot 01$ \\
\hline & $\mathrm{Et}(\mathrm{M})$ & $67 \cdot 6$ & $\mathrm{C}_{13} \mathrm{H}_{24} \mathrm{NNaO}_{4}$ & $1 \cdot 0 \mathrm{H}_{2} \mathrm{O}$ & $52 \cdot 16$ & $8 \cdot 75$ & $4 \cdot 68$ & $52 \cdot 26$ & $8 \cdot 36$ & $4 \cdot 75$ \\
\hline & $\mathrm{n}-\operatorname{Pr}(\mathrm{M})$ & $68 \cdot 1$ & $\mathrm{C}_{14} \mathrm{H}_{26} \mathrm{NNaO}_{4}$ & $1 \cdot 0 \mathrm{H}_{2} \mathrm{O}$ & $53 \cdot 66$ & $9 \cdot 01$ & $4 \cdot 47$ & $54 \cdot 07$ & $8 \cdot 67$ & $4 \cdot 45$ \\
\hline & $\mathrm{i}-\operatorname{Pr}(\mathrm{M})$ & $56 \cdot 0$ & $\mathrm{C}_{14} \mathrm{H}_{26} \mathrm{NNaO}_{4}$ & $1.5 \mathrm{H}_{2} \mathrm{O}$ & $52 \cdot 16$ & $9 \cdot 07$ & $4 \cdot 34$ & $51 \cdot 94$ & $8 \cdot 75$ & $4 \cdot 72$ \\
\hline & $\mathrm{n}-\mathrm{Bu}(\mathrm{A})$ & $75 \cdot 6$ & $\mathrm{C}_{15} \mathrm{H}_{28} \mathrm{NNaO}_{4}$ & $0.7 \mathrm{H}_{2} \mathrm{O}$ & $55 \cdot 96$ & $9 \cdot 20$ & $4 \cdot 35$ & $56 \cdot 08$ & $8 \cdot 92$ & $4 \cdot 27$ \\
\hline & (B) & & $\mathrm{C}_{15} \mathrm{H}_{28} \mathrm{NNaO}_{4}$ & $0.7 \mathrm{H}_{2} \mathrm{O}$ & $55 \cdot 96$ & $9 \cdot 20$ & $4 \cdot 35$ & $55 \cdot 86$ & $9 \cdot 30$ & $4 \cdot 07$ \\
\hline & $\mathrm{i}-\mathrm{Bu}(\mathrm{A})$ & $76 \cdot 5$ & $\mathrm{C}_{15} \mathrm{H}_{28} \mathrm{NNaO}_{4}$ & $1 \cdot 0 \mathrm{H}_{2} \mathrm{O}$ & $55 \cdot 03$ & $9 \cdot 24$ & $4 \cdot 28$ & $55 \cdot 27$ & $9 \cdot 14$ & $4 \cdot 38$ \\
\hline & (B) & & $\mathrm{C}_{15} \mathrm{H}_{28} \mathrm{NNaO}_{4}$ & $1 \cdot 0 \mathrm{H}_{2} \mathrm{O}$ & $55 \cdot 03$ & $9 \cdot 24$ & $4 \cdot 28$ & $55 \cdot 01$ & $9 \cdot 34$ & $4 \cdot 37$ \\
\hline & sec-Bu $(\mathrm{M})$ & $29 \cdot 1$ & $\mathrm{C}_{15} \mathrm{H}_{28} \mathrm{NNaO}_{4}$ & $1 \cdot 4 \mathrm{H}_{2} \mathrm{O}$ & $53 \cdot 84$ & $9 \cdot 28$ & $4 \cdot 19$ & $54 \cdot 12$ & 8.99 & $4 \cdot 52$ \\
\hline & $\left(\mathrm{CH}_{2}\right)_{2} \mathrm{CO}_{2} \mathrm{Na}(\mathrm{M})$ & $66 \cdot 1$ & $\mathrm{C}_{14} \mathrm{H}_{23} \mathrm{NNa}_{2} \mathrm{O}_{6}$ & $2 \cdot 0 \mathrm{H}_{2} \mathrm{O}$ & $43 \cdot 86$ & $7 \cdot 10$ & $3 \cdot 65$ & $44 \cdot 29$ & $6 \cdot 93$ & $3 \cdot 78$ \\
\hline & $\left(\mathrm{CH}_{2}\right)_{3} \mathrm{CO}_{2} \mathrm{Na}(\mathrm{M})$ & $73 \cdot 6$ & $\mathrm{C}_{15} \mathrm{H}_{25} \mathrm{NNa}_{2} \mathrm{O}_{6}$ & $3 \cdot 5 \mathrm{H}_{2} \mathrm{O}$ & $42 \cdot 45$ & $7 \cdot 60$ & $3 \cdot 30$ & $42 \cdot 10$ & $7 \cdot 63$ & $3 \cdot 32$ \\
\hline $\mathrm{n}-\mathrm{Bu}$ & $\mathrm{H}$ & $62 \cdot 5$ & $\mathrm{C}_{12} \mathrm{H}_{22} \mathrm{NNaO}_{4}$ & $1 \cdot 0 \mathrm{H}_{2} \mathrm{O}$ & $50 \cdot 52$ & $8 \cdot 48$ & $4 \cdot 91$ & $50 \cdot 62$ & $8 \cdot 37$ & $5 \cdot 27$ \\
\hline & $\operatorname{Me}(\mathrm{M})$ & $53 \cdot 5$ & $\mathrm{C}_{13} \mathrm{H}_{24} \mathrm{NNaO}_{4}$ & $1 \cdot 0 \mathrm{H}_{2} \mathrm{O}$ & $52 \cdot 16$ & $8 \cdot 75$ & $4 \cdot 68$ & $52 \cdot 61$ & $8 \cdot 64$ & $4 \cdot 78$ \\
\hline & $\mathrm{Et}(\mathrm{M})$ & $69 \cdot 6$ & $\mathrm{C}_{14} \mathrm{H}_{26} \mathrm{NNaO}_{4}$ & $0.8 \mathrm{H}_{2} \mathrm{O}$ & $54 \cdot 28$ & $8 \cdot 98$ & $4 \cdot 52$ & $54 \cdot 34$ & $9 \cdot 30$ & $4 \cdot 46$ \\
\hline & $\mathrm{n}-\operatorname{Pr}(\mathrm{M})$ & $65 \cdot 5$ & $\mathrm{C}_{15} \mathrm{H}_{28} \mathrm{NNaO}_{4}$ & $0.8 \mathrm{H}_{2} \mathrm{O}$ & $55 \cdot 64$ & $9 \cdot 21$ & $4 \cdot 44$ & $55 \cdot 76$ & $9 \cdot 01$ & $4 \cdot 29$ \\
\hline & $\mathrm{i}-\operatorname{Pr}(\mathrm{M})$ & $59 \cdot 3$ & $\mathrm{C}_{15} \mathrm{H}_{28} \mathrm{NNaO}_{4}$ & $1 \cdot 1 \mathrm{H}_{2} \mathrm{O}$ & $54 \cdot 73$ & $9 \cdot 25$ & $4 \cdot 25$ & $54 \cdot 89$ & $8 \cdot 72$ & $4 \cdot 40$ \\
\hline & $\mathrm{n}-\mathrm{Bu}(\mathrm{M})$ & $74 \cdot 3$ & $\mathrm{C}_{16} \mathrm{H}_{30} \mathrm{NNaO}_{4}$ & $0.5 \mathrm{H}_{2} \mathrm{O}$ & $57 \cdot 81$ & $9 \cdot 40$ & $4 \cdot 21$ & $57 \cdot 80$ & $9 \cdot 19$ & $4 \cdot 16$ \\
\hline & $\mathrm{i}-\mathrm{Bu}(\mathrm{M})$ & $75 \cdot 5$ & $\mathrm{C}_{16} \mathrm{H}_{30} \mathrm{NNaO}_{4}$ & $0.5 \mathrm{H}_{2} \mathrm{O}$ & $57 \cdot 81$ & $9 \cdot 40$ & $4 \cdot 21$ & $57 \cdot 75$ & $9 \cdot 04$ & $4 \cdot 14$ \\
\hline & sec-Bu $(\mathrm{M})$ & $27 \cdot 3$ & $\mathrm{C}_{16} \mathrm{H}_{30} \mathrm{NNaO}_{4}$ & $1 \cdot 0 \mathrm{H}_{2} \mathrm{O}$ & $56 \cdot 29$ & $9 \cdot 45$ & $4 \cdot 10$ & $56 \cdot 23$ & $9 \cdot 06$ & $4 \cdot 20$ \\
\hline & $\left(\mathrm{CH}_{2}\right)_{2} \mathrm{CO}_{2} \mathrm{Na}(\mathrm{M})$ & $61 \cdot 9$ & $\mathrm{C}_{15} \mathrm{H}_{15} \mathrm{NNa}_{2} \mathrm{O}_{6}$ & $1 \cdot 0 \mathrm{H}_{2} \mathrm{O}$ & $47 \cdot 49$ & $7 \cdot 17$ & $3 \cdot 69$ & $47 \cdot 51$ & $6 \cdot 91$ & $3 \cdot 77$ \\
\hline & $\left(\mathrm{CH}_{2}\right)_{3} \mathrm{CO}_{2} \mathrm{Na}(\mathrm{M})$ & $62 \cdot 9$ & $\mathrm{C}_{16} \mathrm{H}_{27} \mathrm{NNa}_{2} \mathrm{O}_{6}$ & $1.5 \mathrm{H}_{2} \mathrm{O}$ & $47 \cdot 76$ & $7 \cdot 51$ & $3 \cdot 48$ & $47 \cdot 98$ & $7 \cdot 38$ & $3 \cdot 53$ \\
\hline $\mathrm{i}-\mathrm{Bu}$ & $\mathrm{H}$ & $63 \cdot 8$ & $\mathrm{C}_{12} \mathrm{H}_{22} \mathrm{NNaO}_{4}$ & $0.5 \mathrm{H}_{2} \mathrm{O}$ & $52 \cdot 16$ & $8 \cdot 39$ & $5 \cdot 07$ & $51 \cdot 73$ & $8 \cdot 15$ & 4.91 \\
\hline & $\operatorname{Me}(\mathrm{M})$ & $54 \cdot 3$ & $\mathrm{C}_{13} \mathrm{H}_{24} \mathrm{NNaO}_{4}$ & $1.0 \mathrm{H}_{2} \mathrm{O}$ & $52 \cdot 16$ & $8 \cdot 75$ & $4 \cdot 68$ & $52 \cdot 15$ & $8 \cdot 56$ & $4 \cdot 52$ \\
\hline & Et $(\mathrm{M})$ & $70 \cdot 1$ & $\mathrm{C}_{14} \mathrm{H}_{26} \mathrm{NNaO}_{4}$ & $1 \cdot 0 \mathrm{H}_{2} \mathrm{O}$ & $53 \cdot 66$ & $9 \cdot 01$ & $4 \cdot 47$ & $53 \cdot 45$ & $8 \cdot 79$ & $4 \cdot 58$ \\
\hline & $\mathrm{n}-\operatorname{Pr}(\mathrm{M})$ & $68 \cdot 9$ & $\mathrm{C}_{15} \mathrm{H}_{28} \mathrm{NNaO}_{4}$ & $1 \cdot 0 \mathrm{H}_{2} \mathrm{O}$ & $55 \cdot 03$ & $9 \cdot 24$ & $4 \cdot 28$ & $55 \cdot 43$ & $9 \cdot 10$ & $4 \cdot 32$ \\
\hline & $\mathrm{i}-\operatorname{Pr}(\mathrm{M})$ & $61 \cdot 5$ & $\mathrm{C}_{15} \mathrm{H}_{28} \mathrm{NNaO}_{4}$ & $1 \cdot 0 \mathrm{H}_{2} \mathrm{O}$ & $55 \cdot 03$ & $9 \cdot 24$ & $4 \cdot 28$ & $55 \cdot 03$ & $9 \cdot 20$ & $4 \cdot 41$ \\
\hline
\end{tabular}


Table 2 (continued).

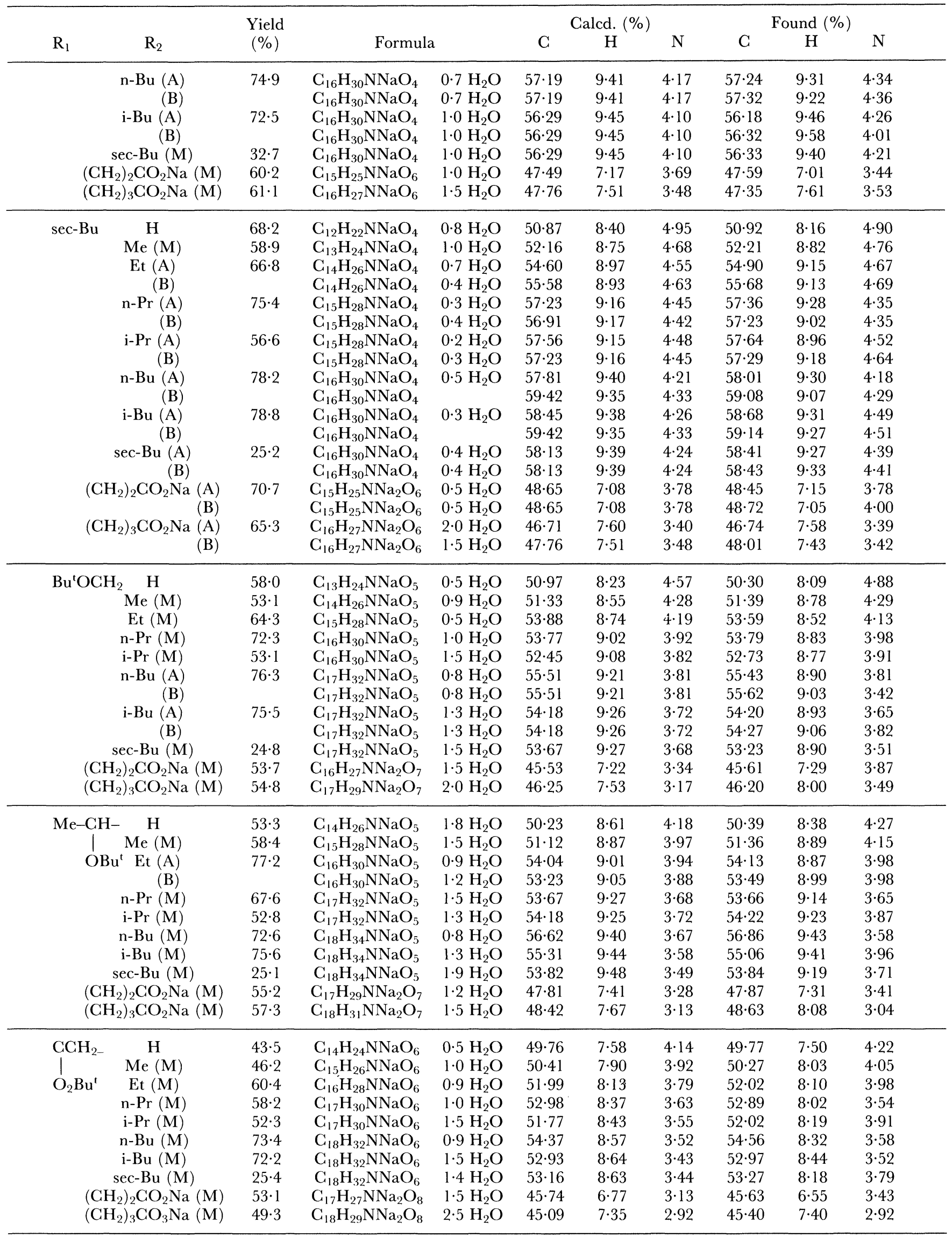

$\mathrm{R}_{1}: \mathrm{R}_{1}-\mathrm{CH}\left(\mathrm{NH}_{2}\right) \mathrm{COOH}, \mathrm{R}_{2}: \mathrm{R}_{2}-\mathrm{COCOOH}, \mathrm{A}$ and $\mathrm{B}$ : separated diastereoisomers, Yield: the combined yield of diastereoisomers, M: a mixture of $\mathrm{A}$ and $\mathrm{B}$. 
Table 3. NMR spectra of substituted $N$-(carboxyalkyl) aminobutyric acid tert-butyl esters.

\begin{tabular}{|c|c|c|c|c|}
\hline $\mathrm{R}$ & $\mathrm{Bu}^{\mathrm{t}}$ & $-\mathrm{CH}-$ & -NHCHR & Other protons (Et and R) \\
\hline $\mathrm{H}$ & $1.49(\mathrm{~s})$ & \multicolumn{2}{|c|}{$\begin{array}{c}3 \cdot 19,3 \cdot 22 \\
(\mathrm{dd}, \mathrm{J}=5 \cdot 86,6 \cdot 85) 2 \mathrm{H}, \mathrm{dd}, \mathrm{J}=16 \cdot 5,18 \cdot 6)\end{array}$} & $0.91(3 \mathrm{H}, \mathrm{t}, \mathrm{J}=7.51), 1.62-1.79(2 \mathrm{H}, \mathrm{m})$ \\
\hline $\mathrm{Me}(\mathrm{M})$ & $\begin{array}{l}1 \cdot 48(\mathrm{~s}) \\
1 \cdot 49(\mathrm{~s})\end{array}$ & \multicolumn{2}{|c|}{$\begin{array}{c}3 \cdot 06-3 \cdot 27 \\
(2 \mathrm{H}, \mathrm{m})\end{array}$} & $\begin{array}{l}0.90,0.92(3 \mathrm{H}, \text { each } t, J=7 \cdot 51), 1 \cdot 216,1.224 \\
(3 \mathrm{H}, \text { each } \mathrm{d}, \mathrm{J}=6.93), 1 \cdot 58-1 \cdot 78(2 \mathrm{H}, \mathrm{c})\end{array}$ \\
\hline Et $(A)$ & $1 \cdot 49(\mathrm{~s})$ & $\begin{array}{c}3 \cdot 09 \\
(\mathrm{t}, \mathrm{J}=6 \cdot 69)\end{array}$ & $\begin{array}{c}2 \cdot 94,2 \cdot 97 \\
(\mathrm{dd}, \mathrm{J}=5 \cdot 77,7 \cdot 42)\end{array}$ & $\begin{array}{l}0.89(3 \mathrm{H}, \mathrm{t}, \mathrm{J}=7 \cdot 42), 0.92(3 \mathrm{H}, \mathrm{t}, \mathrm{J}=7.59) \\
1.50-1.78(4 \mathrm{H}, \mathrm{m})\end{array}$ \\
\hline (B) & $1 \cdot 48(\mathrm{~s})$ & $\begin{array}{c}3 \cdot 17,3 \cdot 19 \\
(\mathrm{dd}, \mathrm{J}=5 \cdot 12,7 \cdot 26)\end{array}$ & $\begin{array}{c}2 \cdot 99,3 \cdot 02 \\
(\mathrm{dd}, \mathrm{J}=6 \cdot 03,6 \cdot 85)\end{array}$ & $\begin{array}{l}0.89(3 \mathrm{H}, \mathrm{t}, \mathrm{J}=7.42), 0.90(3 \mathrm{H}, \mathrm{t}, \mathrm{J}=7.42) \\
1.52-1.78(4 \mathrm{H}, \mathrm{m})\end{array}$ \\
\hline $\mathrm{n}-\operatorname{Pr}(\mathrm{A})$ & $1.49(\mathrm{~s})$ & $\begin{array}{c}3.05 \\
(\mathrm{t}, \mathrm{J}=6.93)\end{array}$ & $\begin{array}{c}3 \cdot 01 \\
(\mathrm{t}, \mathrm{J}=6 \cdot 60)\end{array}$ & $\begin{array}{l}0.91(3 \mathrm{H}, \mathrm{t}, \mathrm{J}=7 \cdot 10), 0.92(3 \mathrm{H}, \mathrm{t}, \mathrm{J}=6.93) \\
1.24-1.42(2 \mathrm{H}, \mathrm{m}), 1.59-1.76(2 \mathrm{H}, \mathrm{m}), 1.43-1.59 \\
(2 \mathrm{H}, \mathrm{m})\end{array}$ \\
\hline (B) & $1 \cdot 48(\mathrm{~s})$ & $\begin{array}{c}3 \cdot 17,3 \cdot 19 \\
(\mathrm{dd}, \mathrm{J}=5 \cdot 11,7 \cdot 26)\end{array}$ & $\begin{array}{l}3 \cdot 06 \\
(\mathrm{t}, \mathrm{J}=6 \cdot 43)\end{array}$ & $\begin{array}{l}0.90(3 \mathrm{H}, \mathrm{t}, \mathrm{J}=7 \cdot 43), 0.91(3 \mathrm{H}, \mathrm{t}, \mathrm{J}=7 \cdot 26) \\
1.62-1.81 \quad(2 \mathrm{H}, \mathrm{m}), 1.24-1.42 \quad(2 \mathrm{H}, \mathrm{m}), 1.47-1.62 \\
(2 \mathrm{H}, \mathrm{m})\end{array}$ \\
\hline $\mathrm{i}-\operatorname{Pr}(\mathrm{A})$ & $1 \cdot 50(\mathrm{~s})$ & $\begin{array}{c}3 \cdot 02 \\
(t, J=7 \cdot 01)\end{array}$ & $\begin{array}{c}2 \cdot 76 \\
(\mathrm{~d}, \mathrm{~J}=5 \cdot 94)\end{array}$ & $\begin{array}{l}0.92,0.95(6 \mathrm{H}, \text { each } \mathrm{d}, \mathrm{J}=6.92), 0.93(3 \mathrm{H}, \mathrm{t} \\
\mathrm{J}=7.51), 1.59-1.75(2 \mathrm{H}, \mathrm{m}), 1.75-1.90(1 \mathrm{H}, \mathrm{m})\end{array}$ \\
\hline (B) & $1 \cdot 48(\mathrm{~s})$ & $\begin{array}{c}3 \cdot 13,3 \cdot 15 \\
(\mathrm{dd}, \mathrm{J}=4 \cdot 95,9 \cdot 42)\end{array}$ & $\begin{array}{c}2 \cdot 83 \\
(d, J=5 \cdot 61)\end{array}$ & $\begin{array}{l}0.90(3 \mathrm{H}, \mathrm{t}, \mathrm{J}=7.67), 0.91,0.93(6 \mathrm{H}, \text { each } \\
\mathrm{d}, \mathrm{J}=6.92), 1.58-1.79(2 \mathrm{H}, \mathrm{m}), 1.79-1.94(1 \mathrm{H}, \mathrm{m})\end{array}$ \\
\hline $\mathrm{n}-\mathrm{Bu}(\mathrm{A})$ & $1 \cdot 50(\mathrm{~s})$ & $\begin{array}{c}3.09 \\
(\mathrm{t}, \mathrm{J}=6 \cdot 77)\end{array}$ & $\begin{array}{c}3 \cdot 03 \\
(\mathrm{t}, \mathrm{J}=6 \cdot 60)\end{array}$ & $\begin{array}{l}0.88(3 \mathrm{H}, \mathrm{t}, \mathrm{J}=7 \cdot 10), 0 \cdot 92(3 \mathrm{H}, \mathrm{t}, \mathrm{J}=7 \cdot 59) \\
1 \cdot 23-1 \cdot 38(4 \mathrm{H}, \mathrm{m}), 1 \cdot 50-1 \cdot 75(4 \mathrm{H}, \mathrm{m})\end{array}$ \\
\hline (B) & $1 \cdot 48(\mathrm{~s})$ & $\begin{array}{c}3 \cdot 18,3 \cdot 21 \\
(\mathrm{dd}, J=5 \cdot 12,7 \cdot 26)\end{array}$ & $\begin{array}{c}3.05 \\
(t, J=6.43)\end{array}$ & $\begin{array}{l}0.89(3 \mathrm{H}, \mathrm{t}, \mathrm{J}=6 \cdot 85), 0.90(3 \mathrm{H}, \mathrm{t}, \mathrm{J}=7 \cdot 51) \\
1 \cdot 20-1 \cdot 35(4 \mathrm{H}, \mathrm{m}), 1 \cdot 55-1 \cdot 80(4 \mathrm{H}, \mathrm{m})\end{array}$ \\
\hline $\mathrm{i}-\mathrm{Bu}(\mathrm{A})$ & $1 \cdot 50(\mathrm{~s})$ & $\begin{array}{c}3.063 \cdot 08 \\
(\mathrm{dd}, \mathrm{J}=5 \cdot 12,6 \cdot 77)\end{array}$ & $\begin{array}{c}3.04 \\
(\mathrm{t}, \mathrm{J}=6 \cdot 19)\end{array}$ & $\begin{array}{l}0.91,0.92(6 \mathrm{H}, \text { each d, } \mathrm{J}=6.43), 0.92(3 \mathrm{H}, \mathrm{t} \\
\mathrm{J}=7.26), 1.40(2 \mathrm{H}, \mathrm{t}, \mathrm{J}=6.85), 1.54-1.75(3 \mathrm{H}, \mathrm{m})\end{array}$ \\
\hline (B) & $1 \cdot 48(\mathrm{~s})$ & $\begin{array}{c}3 \cdot 16,3 \cdot 18 \\
(\mathrm{dd}, \mathrm{J}=5 \cdot 03,7 \cdot 34)\end{array}$ & $\begin{array}{c}3.09 \\
(t, J=7 \cdot 10)\end{array}$ & $\begin{array}{l}0.90(3 \mathrm{H}, \mathrm{t}, \mathrm{J}=7.59), 0.92(6 \mathrm{H}, \mathrm{d}, \mathrm{J}=6.59) \\
1.35-1.53(2 \mathrm{H}, \mathrm{m}), 1.55-1.80(3 \mathrm{H}, \mathrm{m})\end{array}$ \\
\hline sec-Bu (A) & $1 \cdot 49(\mathrm{~s})$ & $\begin{array}{c}3 \cdot 13 \\
(\mathrm{t}, \mathrm{J}=6 \cdot 93)\end{array}$ & $\begin{array}{l}2 \cdot 84(\mathrm{~d}, \mathrm{~J}=5 \cdot 77) \\
2 \cdot 88(\mathrm{~d}, \mathrm{~J}=4 \cdot 61)\end{array}$ & $0 \cdot 85-0.95(9 \mathrm{H}, \mathrm{m}), 1 \cdot 10-1 \cdot 75(5 \mathrm{H}, \mathrm{m})$ \\
\hline (B) & $1 \cdot 48(\mathrm{~s})$ & $\begin{array}{l}3 \cdot 16(t, J=6 \cdot 52) \\
3 \cdot 19(t, J=6 \cdot 44)\end{array}$ & $\begin{array}{l}2 \cdot 92(\mathrm{~d}, \mathrm{~J}=5 \cdot 45) \\
2 \cdot 98(\mathrm{~d}, \mathrm{~J}=4 \cdot 45)\end{array}$ & $0 \cdot 85-0 \cdot 95(9 \mathrm{H}, \mathrm{m})), 1 \cdot 10-1 \cdot 75(5 \mathrm{H}, \mathrm{m})$ \\
\hline$\left(\mathrm{CH}_{2}\right)_{2-}$ & $1 \cdot 48(\mathrm{~s})$ & $3 \cdot 10(\mathrm{t}, \mathrm{J}=6 \cdot 68)$ & $3.00(\mathrm{t}, \mathrm{J}=6 \cdot 68)$ & $0 \cdot 90,0 \cdot 92(3 \mathrm{H}$, each $\mathrm{t}, \mathrm{J}=7 \cdot 43,7 \cdot 51)$ \\
\hline $\mathrm{CO}_{2} \mathrm{Na}(\mathrm{M})$ & $1 \cdot 49(\mathrm{~s})$ & $\begin{array}{c}3 \cdot 18,3 \cdot 20 \\
(\mathrm{dd}, \mathrm{J}=5 \cdot 20,76 \cdot 18)\end{array}$ & $3.04(\mathrm{t}, \mathrm{J}=6.52)$ & $1 \cdot 60-1 \cdot 90(4 \mathrm{H}, \mathrm{c}), 2 \cdot 30-2 \cdot 50(2 \mathrm{H}, \mathrm{c})$ \\
\hline $\begin{array}{l}\left(\mathrm{CH}_{2}\right)_{3}- \\
\mathrm{CO}_{2} \mathrm{Na}(\mathrm{M})\end{array}$ & $\begin{array}{l}1 \cdot 48(\mathrm{~s}) \\
1 \cdot 49(\mathrm{~s})\end{array}$ & $2 \cdot 96-3 \cdot$ & $(2 \mathrm{H}, \mathrm{m})$ & $\begin{array}{l}0 \cdot 90,0 \cdot 92(3 \mathrm{H}, \text { each } t, J=6 \cdot 85,6 \cdot 77) \\
1 \cdot 40-1 \cdot 80(6 \mathrm{H}, \mathrm{c}), 2 \cdot 14-2 \cdot 24(2 \mathrm{H}, \mathrm{c})\end{array}$ \\
\hline
\end{tabular}

the calculated yield presuming consumption of reducing agent. The fractions collected at these two peaks were transferred to the freeze-drying unit, but the fraction eluting from 54.8 to 58.0 min was not collected. Thus the combined synthetic yield of both isomers of 6 was $72 \cdot 5 \%$. A variety of substituted $\mathrm{N}$-(carboxyalkyl)amino-acid tritbutyl ester derivatives (90 compounds) were automatically synthesized in a similar manner to that described above, and the yields with the elemental analysis are presented in table 2. The ${ }^{1} \mathrm{H}$ NMR data are shown in tables 3 to 11 . Since no significant influence from the amino-acid substituents was found, the addition rate of sodium cyanoborohydride was computed using the substituent constants of the 2-keto acids. However, the forward rate constants for glyoxylic acid and pyruvic acid were sufficiently large, in these case, for the reducing agent to be added to the reaction mixture in an uncontrolled process.

\section{Configurational assignment of the products}

The natural L-amino-acids, except for those containing sulphur (cysteine and methionine), have S-configuration on the chiral centre. Thus the reaction of an L-amino-acid tert-butyl ester with a 2-keto acid generally gives two diastereoisomers, $[\mathrm{S}, \mathrm{R}]-$ and $[\mathrm{S}, \mathrm{S}]$-isomers. All of the diastereoisomers derived from the 2-keto acids, except those from glyoxylic acid, pyruvic acid and 2-keto dicarboxylic acid, were separated from each other in the purification unit of the synthesis apparatus. Subsequently, the configurations of the newly formed chiral centres were determined. As a typical example, each diastereoisomer of 6 was derivatized into its $\mathrm{N}$-(carboxy3-methyl)-butyl-L-leucine disodium salt (7) having symmetrical structure. The first eluted peak $(6-a)$ of 6 , converted into the corresponding disodium salt ( $7-a)$ by deblocking with trifluoroacetic acid and purified on a Sephadex LH-20 column, gave the meso-compound having no $[\alpha]_{\mathrm{D}}$ value, whereas the disodium salt $(7-b)$ derived from the second eluted peak (6-b) showed a positive $[\alpha]_{\mathrm{D}}$ value $\left(+39 \cdot 2^{\circ}\right)$. Thus the configuration of the newly formed chiral centre for 6-a was determined as $\mathrm{R}$, and that of $6-\mathrm{b}$ as $\mathrm{S}$.

Typical CD and $[\alpha]_{D}$ of substituted $\mathrm{N}$-(carboxyalkyl)- 
Table 4. NMR spectra of substituted $N$-(carboxyalkyl)norvaline tert-butyl esters.

\begin{tabular}{|c|c|c|c|c|}
\hline $\mathrm{R}$ & $\mathrm{Bu}^{\mathrm{t}}$ & $-\mathrm{CH}-$ & -NHCHR & Other protons \\
\hline $\mathrm{H}$ & $1 \cdot 49(\mathrm{~s})$ & $3 \cdot 26(t, J=6 \cdot 60)$ & $\begin{array}{c}3 \cdot 16,3 \cdot 17 \\
(2 \mathrm{H}, \mathrm{dd}, \mathrm{J}=16 \cdot 5,18 \cdot 5)\end{array}$ & $\begin{array}{l}0 \cdot 92(3 \mathrm{H}, \mathrm{t}, \mathrm{J}=7 \cdot 34), 1 \cdot 25-1 \cdot 43(2 \mathrm{H}, \mathrm{m}) \\
1 \cdot 59-1 \cdot 74(2 \mathrm{H}, \mathrm{m})\end{array}$ \\
\hline $\operatorname{Me}(\mathrm{M})$ & $\begin{array}{l}1.48(\mathrm{~s}) \\
1.49(\mathrm{~s})\end{array}$ & $\begin{array}{l}3 \cdot 21(\mathrm{t}, \mathrm{J}=6 \cdot 76) \\
3 \cdot 26(\mathrm{t}, \mathrm{J}=6 \cdot 52)\end{array}$ & $\begin{array}{c}3 \cdot 10,3 \cdot 11 \\
(\text { each } \mathrm{q}, \mathrm{J}=6 \cdot 93)\end{array}$ & $\begin{array}{l}0.92(3 \mathrm{H}, \mathrm{t}, \mathrm{J}=7 \cdot 26), 1 \cdot 21,1.22(3 \mathrm{H}, \text { each } \mathrm{d}, \\
\mathrm{J}=6.93), 1 \cdot 26-1 \cdot 44(2 \mathrm{H}, \mathrm{c}), 1 \cdot 56-1.83(2 \mathrm{H}, \mathrm{c})\end{array}$ \\
\hline Et (A) & $1.49(\mathrm{~s})$ & $3 \cdot 17(t, J=6 \cdot 85)$ & $\begin{array}{c}2 \cdot 93,2 \cdot 96 \\
(\mathrm{dd}, \mathrm{J}=5 \cdot 78,7 \cdot 43)\end{array}$ & $\begin{array}{l}0.89(3 \mathrm{H}, \mathrm{t}, \mathrm{J}=7 \cdot 43), 0.91(3 \mathrm{H}, \mathrm{t}, \mathrm{J}=7 \cdot 26) \\
1.23-1.44(2 \mathrm{H}, \mathrm{m}), 1.50-1.73(4 \mathrm{H}, \mathrm{m})\end{array}$ \\
\hline Et $(B)$ & $1 \cdot 48(\mathrm{~s})$ & $3 \cdot 23(\mathrm{t}, \mathrm{J}=6 \cdot 36)$ & $3.01(\mathrm{t}, \mathrm{J}=6 \cdot 35)$ & $\begin{array}{l}0.89(3 \mathrm{H}, \mathrm{t}, \mathrm{J}=7.59), 0.91(3 \mathrm{H}, \mathrm{t}, \mathrm{J}=7 \cdot 25), 1.211 .44 \\
(2 \mathrm{H}, \mathrm{m}), \mathrm{l} \cdot 5 \mathrm{l}-\mathrm{l} \cdot 72(4 \mathrm{H}, \mathrm{m})\end{array}$ \\
\hline $\mathrm{n}-\operatorname{Pr}(\mathrm{A})$ & $1 \cdot 49(\mathrm{~s})$ & $3 \cdot 17(\mathrm{t}, \mathrm{J}=6 \cdot 85)$ & $3 \cdot 01(\mathrm{t}, \mathrm{J}=6 \cdot 60)$ & $\begin{array}{l}0.912(3 \mathrm{H}, \mathrm{t}, \mathrm{J}=7.34), 0.905(3 \mathrm{H}, \mathrm{t}, \mathrm{J}=7.26), 1.21- \\
1.42(4 \mathrm{H}, \mathrm{m}), 1.50-1.73(4 \mathrm{H}, \mathrm{m})\end{array}$ \\
\hline n-Pr $(B)$ & $1 \cdot 48(\mathrm{~s})$ & $3 \cdot 23(\mathrm{t}, \mathrm{J}=6 \cdot 36)$ & $3 \cdot 06(\mathrm{t}, \mathrm{J}=6 \cdot 44)$ & $\begin{array}{l}0.907(3 \mathrm{H}, \mathrm{t}, \mathrm{J}=7 \cdot 26), 0.912(3 \mathrm{H}, \mathrm{t}, \mathrm{J}=7.25), 1.21- \\
1.43(4 \mathrm{H}, \mathrm{m}), 1.50-1 \cdot 72(4 \mathrm{H}, \mathrm{m})\end{array}$ \\
\hline $\mathrm{i}-\operatorname{Pr}(\mathrm{A})$ & $1 \cdot 49(\mathrm{~s})$ & $3 \cdot 12(t, J=7 \cdot 01)$ & $2 \cdot 75(\mathrm{~d}, \mathrm{~J}=6 \cdot 10)$ & $\begin{array}{l}0.91(3 \mathrm{H}, \mathrm{t}, \mathrm{J}=7 \cdot 25), 0.92,0.95(6 \mathrm{H}, \text { each } \mathrm{d}, \mathrm{J}=6.92), \\
1.24-1.44(2 \mathrm{H}, \mathrm{m}), 1.53-1.75(2 \mathrm{H}, \mathrm{m}), 1 \cdot 75-1.89(1 \mathrm{H},)\end{array}$ \\
\hline $\mathrm{i}-\operatorname{Pr}(\mathrm{B})$ & $1 \cdot 48(\mathrm{~s})$ & $3 \cdot 18(t, J=6 \cdot 35)$ & $2 \cdot 84(\mathrm{~d}, \mathrm{~J}=5 \cdot 61)$ & $\begin{array}{l}0.91(3 \mathrm{H}, \mathrm{t}, \mathrm{J}=7.26), 0.91,0.93(6 \mathrm{H}, \text { each } \mathrm{d}, \mathrm{J}=6.93) \\
1.20-1.45 \quad(2 \mathrm{H}, \mathrm{m}), \quad 1.58-1.72(2 \mathrm{H}, \mathrm{m}), 1.80-1.94 \\
(1 \mathrm{H}, \mathrm{m})\end{array}$ \\
\hline n-Bu (A) & $1 \cdot 49(\mathrm{~s})$ & $3 \cdot 18(t, J=6 \cdot 85)$ & $3 \cdot 01(t, J=6 \cdot 60)$ & $\begin{array}{l}0.88(3 \mathrm{H}, \mathrm{t}, \mathrm{J}=6.93), 0.91(3 \mathrm{H}, \mathrm{t}, \mathrm{J}=7 \cdot 25), 1.23-1.42 \\
(6 \mathrm{H}, \mathrm{m}), 1.45-1.75(4 \mathrm{H}, \mathrm{m})\end{array}$ \\
\hline n-Bu (B) & $1 \cdot 48(\mathrm{~s})$ & $3 \cdot 22(\mathrm{t}, \mathrm{J}=6 \cdot 36)$ & $3 \cdot 04(\mathrm{t}, \mathrm{J}=6 \cdot 52)$ & $\begin{array}{l}0 \cdot 89(3 \mathrm{H}, \mathrm{t}, \mathrm{J}=6 \cdot 77), 0 \cdot 91(3 \mathrm{H}, \mathrm{t}, \mathrm{J}=7 \cdot 26), 1 \cdot 20-1 \cdot 43 \\
(6 \mathrm{H}, \mathrm{m}), 1 \cdot 50-1 \cdot 72(4 \mathrm{H}, \mathrm{m})\end{array}$ \\
\hline $\mathrm{i}-\mathrm{Bu}(\mathrm{A})$ & $1 \cdot 49(\mathrm{~s})$ & $3 \cdot 15(\mathrm{t}, \mathrm{J}=6 \cdot 85)$ & $3 \cdot 04(t, J=7 \cdot 10)$ & $\begin{array}{l}0.91(3 \mathrm{H}, \mathrm{t}, \mathrm{J}=7 \cdot 26), 0 \cdot 91,0.92(6 \mathrm{H}, \text { each } \mathrm{d}, \mathrm{J}=6 \cdot 59), \\
1 \cdot 25-1 \cdot 45(2 \mathrm{H}, \mathrm{m}), 1 \cdot 39(2 \mathrm{H}, \mathrm{t}-\mathrm{like}, \mathrm{J}=7 \cdot 10), 1 \cdot 55-1 \cdot 75 \\
(3 \mathrm{H}, \mathrm{m})\end{array}$ \\
\hline $\mathrm{i}-\mathrm{Bu}(\mathrm{B})$ & $1 \cdot 48(\mathrm{~s})$ & $3 \cdot 21(t, J=6 \cdot 35)$ & $3.09(\mathrm{t}, \mathrm{J}=7 \cdot 01)$ & $\begin{array}{l}0.91(3 \mathrm{H}, \mathrm{t}, \mathrm{J}=7 \cdot 26), 0.92(6 \mathrm{H}, \mathrm{d}, \mathrm{J}=6.59), 1 \cdot 20-1.50 \\
(4 \mathrm{H}, \mathrm{m}), 1 \cdot 55-1.72(3 \mathrm{H}, \mathrm{m})\end{array}$ \\
\hline sec-Bu (A) & $1 \cdot 50(\mathrm{~s})$ & $3 \cdot 03(t, J=7 \cdot 01)$ & $\begin{array}{l}2 \cdot 84(\mathrm{~d}, \mathrm{~J}=5 \cdot 77) \\
2 \cdot 89(\mathrm{~d}, \mathrm{~J}=4 \cdot 61)\end{array}$ & $\begin{array}{l}0.90(3 \mathrm{H}, \mathrm{t}, \mathrm{J}=7 \cdot 10), 0 \cdot 91(3 \mathrm{H}, \mathrm{d}, \mathrm{J}=7 \cdot 26) \\
0.93(3 \mathrm{H}, \mathrm{t}, \mathrm{J}=7 \cdot 34), 1 \cdot 10-1 \cdot 30(1 \mathrm{H}, \mathrm{m}), 1 \cdot 35-1 \cdot 80 \\
(6 \mathrm{H}, \mathrm{m})\end{array}$ \\
\hline sec-Bu (B) & $1 \cdot 48(\mathrm{~s})$ & $\begin{array}{c}3 \cdot 11,3 \cdot 14 \\
\text { dd, } J=4 \cdot 95,7 \cdot 42) \\
3 \cdot 14,3 \cdot 16 \\
(\mathrm{dd}, \mathrm{J}=5 \cdot 10,6 \cdot 76)\end{array}$ & $\begin{array}{l}2 \cdot 92(\mathrm{~d}, \mathrm{~J}=5 \cdot 28) \\
2 \cdot 98(\mathrm{~d}, \mathrm{~J}=4 \cdot 62)\end{array}$ & $\begin{array}{l}0 \cdot 85-0 \cdot 95(9 \mathrm{H}, \mathrm{m}), 1 \cdot 10-1 \cdot 27(1 \mathrm{H}, \mathrm{m}) \\
1 \cdot 35-1 \cdot 82(6 \mathrm{H}, \mathrm{m})\end{array}$ \\
\hline$\left(\mathrm{CH}_{2}\right)_{2}-$ & $\begin{array}{l}1 \cdot 475(\mathrm{~s}) \\
1 \cdot 489(\mathrm{~s})\end{array}$ & $\begin{array}{l}3 \cdot 17(\mathrm{t}, \mathrm{J}=6 \cdot 85) \\
3 \cdot 23(\mathrm{t}, \mathrm{J}=6 \cdot 44)\end{array}$ & $\begin{array}{l}2 \cdot 99(\mathrm{t}, \mathrm{J}=6 \cdot 68) \\
3 \cdot 03(\mathrm{t}, \mathrm{J}=6 \cdot 43)\end{array}$ & $\begin{array}{l}0.91(3 \mathrm{H}, \mathrm{t}, \mathrm{J}=7 \cdot 34), 1 \cdot 25-1 \cdot 43(2 \mathrm{H}, \mathrm{c}) \\
1 \cdot 50-1 \cdot 88(4 \mathrm{H}, \mathrm{c}), 2 \cdot 13-2 \cdot 27(2 \mathrm{H}, \mathrm{c})\end{array}$ \\
\hline$\left(\mathrm{CH}_{2}\right)_{3-}$ & $1 \cdot 489(\mathrm{~s})$ & $3 \cdot 17(\mathrm{t}, \mathrm{J}=6 \cdot 85)$ & $\begin{array}{c}2 \cdot 99 \\
\text { (undefined } t)\end{array}$ & $\begin{array}{l}0.91(3 \mathrm{H}, \mathrm{t}, \mathrm{J}=7 \cdot 26), 1 \cdot 25-1 \cdot 45(2 \mathrm{H}, \mathrm{m}) \\
1.50-1 \cdot 75(6 \mathrm{H}, \mathrm{m}), 2 \cdot 19(2 \mathrm{H}, \text { undefined } \mathrm{t})\end{array}$ \\
\hline $\begin{array}{l}\mathrm{CO}_{2} \mathrm{Na}(\mathrm{A}) \\
\left(\mathrm{CH}_{2}\right)_{3}- \\
\mathrm{CO}_{2} \mathrm{Na}(\mathrm{B})\end{array}$ & $1 \cdot 490(\mathrm{~s})$ & $3 \cdot 17(\mathrm{t}, \mathrm{J}=6 \cdot 85)$ & $\begin{array}{c}3 \cdot 00 \\
\text { (undefined } t)\end{array}$ & $\begin{array}{l}0.91(3 \mathrm{H}, \mathrm{t}, \mathrm{J}=7 \cdot 26), 1 \cdot 25-1 \cdot 45(2 \mathrm{H}, \mathrm{m}) \\
1 \cdot 50-1 \cdot 75(6 \mathrm{H}, \mathrm{m}), 2 \cdot 19(2 \mathrm{H}, \text { undefined } \mathrm{t})\end{array}$ \\
\hline
\end{tabular}

amino-acid tert-butyl esters are shown in table 12. All of the first eluted products (A) and the second eluted products (B) showed negative Cotton effects at 227-237 $\mathrm{nm}$ and positive Cotton effects at $220-225 \mathrm{~nm}$ in water, respectively.

In the ${ }^{1} \mathrm{H}$ NMR spectra of substituted N-(carboxyalkyl)amino-acid tert-butyl ester, the chemical shift of the tertbutyl ester group (1.48-1.50 ppm) in the A-isomers was found to be generally at lower field (ca. $0.01 \mathrm{ppm}$ ) than in the B-isomers. The chemical shifts of the methine protons on the two chiral centres of the A-isomers (around 2.9-3.6 and $2 \cdot 7-3 \cdot 2 \mathrm{ppm})$ were observed at higher field $(0 \cdot 05-0 \cdot 1$ ppm) than those of the B-isomers.

\section{Conclusion}

The reaction control equations incorporated in a computer program have been applied to the synthesis of substituted $\mathrm{N}$-(carboxyalkyl)amino-acids, and all synthetic processes were automatically performed from the mixing of reactants to the isolation of the products as powders. The automated apparatus was run continuously for 24 hours, giving an average rate of synthesis of three compounds per day. The apparatus extremely valuable for synthesizing many derivatives of a particular compound. Even if the chemical yields are low under the optimum conditions. It is still possible to obtain a sufficient amount of the desired product by repeating the reaction. Moreover, it is possible to greatly reduce the labour-intensive nature of the many syntheses which are a necessary part of pharmaceutical research.

\section{Experimental}

Infra-red and ultraviolet spectra were measured on Hitachi 260-10 and Hitachi 557 double wavelength spectrophotometers, respectively. Optical rotations 
Table 5. NMR spectra of substituted $N$-(carboxyalkyl)valine tert-butyl esters.

\begin{tabular}{|c|c|c|c|c|}
\hline $\mathrm{R}$ & $\mathrm{Bu}^{\mathrm{t}}$ & $-\mathrm{CH}-$ & -NHCHR & Other protons \\
\hline $\mathrm{H}$ & $1 \cdot 495(\mathrm{~s})$ & $3 \cdot 04(\mathrm{~d}, \mathrm{~J}=5 \cdot 77)$ & $\begin{array}{c}3 \cdot 07,3 \cdot 14 \\
(2 \mathrm{H}, \mathrm{dd}, \mathrm{J}=16 \cdot 4,19 \cdot 9)\end{array}$ & $0.97(6 \mathrm{H}, \mathrm{t}, \mathrm{J}=6.85), 1.99(1 \mathrm{H}, \mathrm{sex}, \mathrm{J}=6.67)$ \\
\hline $\operatorname{Me}(\mathrm{M})$ & $\begin{array}{l}1 \cdot 484(\mathrm{~s}) \\
1 \cdot 496(\mathrm{~s})\end{array}$ & $2 \cdot 94(\mathrm{~d}, \mathrm{~J}=6 \cdot 60)$ & $3 \cdot 00-3 \cdot 12(\mathrm{c})$ & $\begin{array}{l}0.90-1.03(6 \mathrm{H}, \mathrm{c}), 1.22(3 \mathrm{H}, \mathrm{d}, \mathrm{J}=6.92) \\
1.90-2 \cdot 05(1 \mathrm{H}, \mathrm{c})\end{array}$ \\
\hline Et $(\mathrm{M})$ & $\begin{array}{l}1.483(\mathrm{~s}) \\
1.499(\mathrm{~s})\end{array}$ & $\begin{array}{l}2 \cdot 89(\mathrm{~d}, \mathrm{~J}=6 \cdot 76) \\
3 \cdot 04(\mathrm{~d}, \mathrm{~J}=5 \cdot 80)\end{array}$ & $\begin{array}{l}2 \cdot 87,2 \cdot 90 \\
(\mathrm{dd}, \mathrm{J}=5 \cdot 61,7 \cdot 75) \\
2.96(\mathrm{t}, \mathrm{J}=6 \cdot 27)\end{array}$ & $0.84-1.02(9 \mathrm{H}, \mathrm{c}), 1.96(1 \mathrm{H}, \mathrm{sep}, \mathrm{J}=6.73)$ \\
\hline $\mathrm{n}-\operatorname{Pr}(\mathrm{M})$ & $\begin{array}{l}1 \cdot 483(\mathrm{~s}) \\
1 \cdot 500(\mathrm{~s})\end{array}$ & $\begin{array}{l}2 \cdot 87(d, J=6 \cdot 93) \\
3 \cdot 03(d, J=5 \cdot 61)\end{array}$ & $\begin{array}{l}2.95(t, J=6.85) \\
3.02(t, J=6.02)\end{array}$ & $\begin{array}{l}0.86-1 \cdot 01(9 \mathrm{H}, \mathrm{c}), 1 \cdot 2-1 \cdot 4(2 \mathrm{H}, \mathrm{c}), 1.4-1.63 \\
(2 \mathrm{H}, \mathrm{c}), 1.95(1 \mathrm{H}, \mathrm{sep}, \mathrm{J}=6.64)\end{array}$ \\
\hline $\mathrm{i}-\operatorname{Pr}(\mathrm{M})$ & $\begin{array}{l}1.480(\mathrm{~s}) \\
1.500(\mathrm{~s})\end{array}$ & $\begin{array}{l}2 \cdot 67(\mathrm{~d}, \mathrm{~J}=6 \cdot 26) \\
2 \cdot 77(\mathrm{~d}, \mathrm{~J}=5 \cdot 61)\end{array}$ & $\begin{array}{l}2 \cdot 78(\mathrm{~d}, \mathrm{~J}=7 \cdot 43) \\
2 \cdot 98(\mathrm{~d}, \mathrm{~J}=5 \cdot 77)\end{array}$ & $0 \cdot 85-1.03(12 \mathrm{H}, \mathrm{c}), 1 \cdot 70-2 \cdot 03(2 \mathrm{H}, \mathrm{c})$ \\
\hline $\mathrm{n}-\mathrm{Bu}(\mathrm{A})$ & $1 \cdot 501(\mathrm{~s})$ & $2 \cdot 87(\mathrm{~d}, \mathrm{~J}=6 \cdot 93)$ & $\begin{array}{l}2 \cdot 92,2 \cdot 95 \\
(\mathrm{dd}, \mathrm{J}=6 \cdot 11,7 \cdot 43)\end{array}$ & $\begin{array}{l}0.89(3 \mathrm{H}, \mathrm{t}, \mathrm{J}=6.93), 0.93,0.97(6 \mathrm{H}, \text { each } \mathrm{d}, \mathrm{J}=6.77) \\
1.23-1.40(4 \mathrm{H}, \mathrm{m}), 1.47-1.65(2 \mathrm{H}, \mathrm{m}), 1.92(1 \mathrm{H}, \mathrm{sex}, \\
\mathrm{J}=6.93)\end{array}$ \\
\hline $\mathrm{n}-\mathrm{Bu}(\mathrm{B})$ & $1 \cdot 483(\mathrm{~s})$ & $3 \cdot 03(\mathrm{~d}, \mathrm{~J}=5 \cdot 61)$ & $2 \cdot 99(\mathrm{t}, \mathrm{J}=6 \cdot 35)$ & $\begin{array}{l}0.89(3 \mathrm{H}, \mathrm{t}, \mathrm{J}=6.93), 0.94,0.98(6 \mathrm{H}, \text { each } \mathrm{d}, \mathrm{J}=6.76, \\
6.92), 1.22-1.38(4 \mathrm{H}, \mathrm{m}), 1.50-1.65(2 \mathrm{H}, \mathrm{m}), 1.97(1 \mathrm{H}, \\
\operatorname{sex}, \mathrm{J}=6.54)\end{array}$ \\
\hline $\mathrm{i}-\mathrm{Bu}(\mathrm{A})$ & $1.505(\mathrm{~s})$ & $2 \cdot 86(\mathrm{~d}, \mathrm{~J}=6 \cdot 93)$ & $2 \cdot 99(t, J=7 \cdot 10)$ & $\begin{array}{l}0.84-1 \cdot 01(12 \mathrm{H}, \mathrm{m}), 1 \cdot 40(2 \mathrm{H}, \mathrm{t}, \mathrm{J}=6 \cdot 85), 1 \cdot 54-1 \cdot 70 \\
(1 \mathrm{H}, \mathrm{m}), 1 \cdot 94(1 \mathrm{H}, \mathrm{sex}, \mathrm{J}=6 \cdot 76)\end{array}$ \\
\hline $\mathrm{i}-\mathrm{Bu}(\mathrm{B})$ & $1 \cdot 485(\mathrm{~s})$ & $3 \cdot 03(\mathrm{~d}, \mathrm{~J}=5 \cdot 45)$ & $3.03(t, J=6.43)$ & $\begin{array}{l}0.91,0.92,0.93,0.98(\text { each } 3 \mathrm{H}, \text { each d, } \mathrm{J}=6.43,6.59, \\
6.93,6.92), 1.37-1.50(2 \mathrm{H}, \mathrm{m}), 1.65(1 \mathrm{H}, \mathrm{sep}, \mathrm{J}=6.77) \text {, } \\
1.96(1 \mathrm{H}, \mathrm{sex}, 6.76)\end{array}$ \\
\hline sec-Bu $(\mathrm{M})$ & $\begin{array}{l}1.476(\mathrm{~s}) \\
1.481(\mathrm{~s}) \\
1.499(\mathrm{~s})\end{array}$ & $2 \cdot 70-3 \cdot 00$ & & $\begin{array}{l}0.80-1 \cdot 03(12 \mathrm{H}, \mathrm{c}), 1 \cdot 0-1.3(1 \mathrm{H}, \mathrm{c}), 1 \cdot 30-1 \cdot 75(2 \mathrm{H}, \mathrm{c}), \\
1.93(1 \mathrm{H}, \text { undefined sex })\end{array}$ \\
\hline 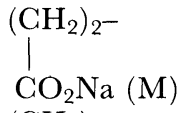 & $\begin{array}{l}1 \cdot 480(\mathrm{~s}) \\
1.500(\mathrm{~s})\end{array}$ & $\begin{array}{l}2 \cdot 89(\mathrm{~d}, \mathrm{~J}=6 \cdot 76) \\
4 \cdot 04(\mathrm{~d}, \mathrm{~J}=5 \cdot 44)\end{array}$ & $\begin{array}{l}2 \cdot 92,2 \cdot 95(\mathrm{dd}, J=6 \cdot 44 \\
7 \cdot 43), 2 \cdot 99(\mathrm{t}, \mathrm{J}=6 \cdot 43)\end{array}$ & $\begin{array}{l}0.93,0.95(3 \mathrm{H}, \text { each, } \mathrm{d}, \mathrm{J}=6.77), 0.976,0.982(3 \mathrm{H}, \\
\text { each } \mathrm{d}, \mathrm{J}=6.93), 1 \cdot 7-1.9(2 \mathrm{H}, \mathrm{c}), 1.96(1 \mathrm{H}, \text { undefined } \\
\text { sep), } 2 \cdot 1-2 \cdot 3(2 \mathrm{H}, \mathrm{c})\end{array}$ \\
\hline $\begin{array}{l}\left(\mathrm{CH}_{2}\right)_{3-}- \\
\mathrm{CO}_{\mathrm{a}} \mathrm{Na}(\mathrm{M})\end{array}$ & $\begin{array}{l}1.481(\mathrm{~s}) \\
1.500(\mathrm{~s})\end{array}$ & $\begin{array}{l}2 \cdot 88(\mathrm{~d}, \mathrm{~J}=6 \cdot 76) \\
3 \cdot 04(\mathrm{~d}, \mathrm{~J}=5 \cdot 44)\end{array}$ & $2 \cdot 90-3 \cdot 07(\mathrm{c})$ & $\begin{array}{l}0.93,0.94,0.97,0.98(6 \mathrm{H}, \text { each } \mathrm{d}, \mathrm{J}=6.93), 1 \cdot 50-1.65 \\
(4 \mathrm{H}, \mathrm{c}), 1.95(1 \mathrm{H}, \text { sep, } \mathrm{J}=6.92), \text { ca. } 2 \cdot 19(2 \mathrm{H}, \\
\text { undefined br. t })\end{array}$ \\
\hline
\end{tabular}

Table 6. NMR spectra of substituted $N$-(carboxyalkyl)norleucine tert-butyl ester.

\begin{tabular}{|c|c|c|c|c|}
\hline $\mathrm{R}$ & $\mathrm{Bu}^{\mathrm{t}}$ & $-\mathrm{CH}-$ & -NHCHR & Other protons \\
\hline $\mathrm{H}$ & $1 \cdot 501(\mathrm{~s})$ & $3 \cdot 25(\mathrm{t}, \mathrm{J}=6 \cdot 52)$ & $\begin{array}{c}3.11,3.17(2 \mathrm{H}, \mathrm{dd}, \mathrm{J}= \\
16 \cdot 5,17 \cdot 8)\end{array}$ & $\begin{array}{l}0.90(3 \mathrm{H}, \mathrm{t}, \mathrm{J}=6.77), 1.23-1.42,(4 \mathrm{H}, \mathrm{m}), 1.60-1.77 \\
(2 \mathrm{H}, \mathrm{m})\end{array}$ \\
\hline $\mathrm{Me}(\mathrm{M})$ & $\begin{array}{l}1.478(\mathrm{~s}) \\
1.486(\mathrm{~s}) 3 \cdot\end{array}$ & $\begin{array}{l}3 \cdot 20(\mathrm{t}, \mathrm{J}=6 \cdot 77) \\
4,3 \cdot 27(\mathrm{dd}, \mathrm{J}=5 \cdot 78 \\
7 \cdot 26)\end{array}$ & $\begin{array}{l}3 \cdot 10(\mathrm{q}, \mathrm{J}=7 \cdot 09) \\
3 \cdot 11(\mathrm{q}, \mathrm{J}=6 \cdot 93)\end{array}$ & $\begin{array}{l}0.89(3 \mathrm{H}, \mathrm{t}, \mathrm{J}=6 \cdot 77), 1 \cdot 21,1 \cdot 22(3 \mathrm{H}, \text { each } \mathrm{d}, \mathrm{J}=6.93 \\
7 \cdot 09), 1 \cdot 22-1 \cdot 39(4 \mathrm{H}, \mathrm{c}), 1 \cdot 57-1 \cdot 75(2 \mathrm{H}, \mathrm{c})\end{array}$ \\
\hline Et $(\mathrm{M})$ & $\begin{array}{l}1 \cdot 478(\mathrm{~s}) \\
1 \cdot 489(\mathrm{~s})\end{array}$ & $\begin{array}{l}3 \cdot 16(\mathrm{t}, \mathrm{J}=6 \cdot 85) \\
3 \cdot 21(\mathrm{t}, \mathrm{J}=6 \cdot 35)\end{array}$ & $\begin{array}{c}2 \cdot 93, .2 \cdot 96(\mathrm{dd}, \mathrm{J}=5 \cdot 86 \\
7 \cdot 18), 3 \cdot 00(\mathrm{t}, \mathrm{J}=6.27)\end{array}$ & $0.89(6 \mathrm{H}, \mathrm{t}, \mathrm{J}=7 \cdot 5 \mathrm{l}), 1 \cdot 2-1 \cdot 4(2 \mathrm{H}, \mathrm{c}), 1 \cdot 5-1 \cdot 74(6 \mathrm{H}, \mathrm{c})$ \\
\hline $\mathrm{n}-\operatorname{Pr}(\mathrm{M})$ & $\begin{array}{l}1.477(\mathrm{~s}) \\
1.490(\mathrm{~s})\end{array}$ & $\begin{array}{l}3 \cdot 15(\mathrm{t}, \mathrm{J}=6 \cdot 77) \\
3 \cdot 21(\mathrm{t}, \mathrm{J}=6 \cdot 35)\end{array}$ & $\begin{array}{c}3 \cdot 00,3 \cdot 05 \\
(\text { each } t, J=6 \cdot 60)\end{array}$ & $0 \cdot 83-0.96(6 \mathrm{H}, \mathrm{c}), 1 \cdot 2-1 \cdot 4(6 \mathrm{H}, \mathrm{c}), 1 \cdot 41-1 \cdot 75(4 \mathrm{H}, \mathrm{c})$ \\
\hline $\mathrm{i}-\operatorname{Pr}(\mathrm{M})$ & $\begin{array}{l}1.476(\mathrm{~s}) \\
1.491(\mathrm{~s})\end{array}$ & $\begin{array}{l}3.09(\mathrm{t}, \mathrm{J}=7 \cdot 02) \\
3 \cdot 17(\mathrm{t}, \mathrm{J}=6 \cdot 36)\end{array}$ & $\begin{array}{l}2 \cdot 74(\mathrm{~d}, \mathrm{~J}=5 \cdot 93) \\
2 \cdot 83(\mathrm{~d}, \mathrm{~J}=5 \cdot 61)\end{array}$ & $\begin{array}{l}0.82-1.00(9 \mathrm{H}, \mathrm{c}), 1.2-1.4(4 \mathrm{H}, \mathrm{c}), 1.55-1.75(2 \mathrm{H}, \mathrm{c}), \\
1.75-1.94(1 \mathrm{H}, \mathrm{c})\end{array}$ \\
\hline $\mathrm{n}-\mathrm{Bu}(\mathrm{M})$ & $\begin{array}{l}1.477(\mathrm{~s}) \\
1.490(\mathrm{~s})\end{array}$ & $\begin{array}{l}3 \cdot 15(\mathrm{t}, \mathrm{J}=6 \cdot 85) \\
3 \cdot 21(\mathrm{t}, \mathrm{J}=6 \cdot 27)\end{array}$ & $\begin{array}{l}2.99(t, J=6.52) \\
3.03(t, J=6.27)\end{array}$ & $0.81-0.95(6 \mathrm{H}, \mathrm{c}), 1.2-1.4(8 \mathrm{H}, \mathrm{c}), 1.45-1.75(4 \mathrm{H}, \mathrm{c})$ \\
\hline $\mathrm{i}-\mathrm{Bu}(\mathrm{M})$ & $\begin{array}{l}1.480(\mathrm{~s}) \\
1.495(\mathrm{~s})\end{array}$ & $\begin{array}{l}3 \cdot 14(t, J=6 \cdot 93) \\
3 \cdot 21(t, J=6 \cdot 35)\end{array}$ & $\begin{array}{l}3 \cdot 04(t, J=6 \cdot 19) \\
3 \cdot 09(t, J=6 \cdot 60)\end{array}$ & $0 \cdot 82-0.96(9 \mathrm{H}, \mathrm{c}), 1 \cdot 2-1 \cdot 75(9 \mathrm{H}, \mathrm{c})$ \\
\hline sec-Bu $(\mathrm{M})$ & $\begin{array}{l}1.475(\mathrm{~s}) \\
1.489(\mathrm{~s})\end{array}$ & $3 \cdot 02-3 \cdot 22(\mathrm{~s})$ & $\begin{array}{l}2 \cdot 82(\mathrm{~d}, \mathrm{~J}=5 \cdot 77) \\
2 \cdot 87(\mathrm{~d}, \mathrm{~J}=4 \cdot 62) \\
2 \cdot 90(\mathrm{~d}, \mathrm{~J}=5 \cdot 28) \\
2 \cdot 97(\mathrm{~d}, \mathrm{~J}=4 \cdot 29)\end{array}$ & $0 \cdot 82-0.97(9 \mathrm{H}, \mathrm{c}), 1 \cdot 10-1 \cdot 75(9 \mathrm{H}, \mathrm{c})$ \\
\hline$\left(\mathrm{CH}_{2}\right)_{2}-$ & $\begin{array}{l}1.476(\mathrm{~s}) \\
1.490(\mathrm{~s})\end{array}$ & $\begin{array}{l}3 \cdot 16(\mathrm{t}, \mathrm{J}=6 \cdot 77) \\
3 \cdot 23(\mathrm{t}, \mathrm{J}=6 \cdot 36)\end{array}$ & $\begin{array}{l}2.99(\mathrm{t}, \mathrm{J}=6 \cdot 60) \\
3.04(\mathrm{t}, \mathrm{J}=6 \cdot 19)\end{array}$ & $\begin{array}{l}0 \cdot 89(3 \mathrm{H}, \mathrm{t}, \mathrm{J}=7 \cdot 10), 1 \cdot 22-1 \cdot 40(4 \mathrm{H}, \mathrm{c}), 1 \cdot 56-1 \cdot 88 \\
(4 \mathrm{H}, \mathrm{c}), 2 \cdot 14-2 \cdot 26(2 \mathrm{H}, \mathrm{c})\end{array}$ \\
\hline $\begin{array}{l}\mathrm{CO}_{2} \mathrm{Na}(\mathrm{M}) \\
\left(\mathrm{CH}_{2}\right)_{3-} \\
\mathrm{CO}_{2} \mathrm{Na}(\mathrm{M})\end{array}$ & $\begin{array}{l}1.476(\mathrm{~s}) \\
1.489(\mathrm{~s})\end{array}$ & $\begin{array}{l}3 \cdot 15(\mathrm{t}, \mathrm{J}=6 \cdot 85) \\
3 \cdot 22(\mathrm{t}, \mathrm{J}=6 \cdot 36)\end{array}$ & $2 \cdot 94-3.09(\mathrm{c})$ & $\begin{array}{l}0 \cdot 89(3 \mathrm{H}, \mathrm{t}, \mathrm{J}=6 \cdot 69), 1 \cdot 2-1 \cdot 4(4 \mathrm{H}, \mathrm{c}), 1 \cdot 50-1 \cdot 75(6 \mathrm{H}, \\
\text { c), } 2 \cdot 19(2 \mathrm{H}, \mathrm{c})\end{array}$ \\
\hline
\end{tabular}


Table 7. NMR spectra of substituted $N$-(carboxyalkyl)leucine tert-butyl esters.

\begin{tabular}{|c|c|c|c|c|}
\hline $\mathrm{R}$ & $\mathrm{Bu}^{\mathrm{t}}$ & $-\mathrm{CH}-$ & -NHCHR & Other protons \\
\hline $\mathrm{H}$ & $1 \cdot 484(\mathrm{~s})$ & $\begin{array}{c}3 \cdot 25,3 \cdot 28(\mathrm{dd}, \mathrm{J}=6 \cdot 43 \\
7 \cdot 92)\end{array}$ & $3 \cdot 12(2 \mathrm{H}, \mathrm{br} . \mathrm{s})$ & $\begin{array}{l}0.92(3 \mathrm{H}, \mathrm{d}, \mathrm{J}=6.27), 0.94(3 \mathrm{H}, \mathrm{d}, \mathrm{J}=6.43), 1.40- \\
1.75(3 \mathrm{H}, \mathrm{m})\end{array}$ \\
\hline $\mathrm{Me}(\mathrm{M})$ & $\begin{array}{l}1.475(\mathrm{~s}) \\
1.481(\mathrm{~s})\end{array}$ & $3 \cdot 22-3 \cdot 32(\mathrm{c})$ & $3 \cdot 02-3 \cdot 16(c)$ & $\begin{array}{l}0.85-0.95(6 \mathrm{H}, \mathrm{c}), 1 \cdot 21,1.22(3 \mathrm{H}, \text { each } \mathrm{d}, \mathrm{J}=6.93), \\
1.30-1.70(3 \mathrm{H}, \mathrm{c})\end{array}$ \\
\hline Et $(\mathrm{M})$ & $\begin{array}{l}1.474(\mathrm{~s}) \\
1.486(\mathrm{~s})\end{array}$ & $3 \cdot 18-3 \cdot 29(\mathrm{c})$ & $\begin{array}{l}2 \cdot 92,2 \cdot 94(\mathrm{dd}, \mathrm{J}=5 \cdot 86 \\
7 \cdot 34), 3 \cdot 01(\mathrm{t}, \mathrm{J}=6 \cdot 27)\end{array}$ & $0.83-0.97(9 \mathrm{H}, \mathrm{c}), 1.4-1.7(5 \mathrm{H}, \mathrm{c})$ \\
\hline $\mathrm{n}-\operatorname{Pr}(\mathrm{M})$ & $\begin{array}{l}1.473(\mathrm{~s}) \\
1.487(\mathrm{~s})\end{array}$ & $3 \cdot 17-3 \cdot 28(\mathrm{c})$ & $\begin{array}{l}2.99(\mathrm{t}, \mathrm{J}=6 \cdot 60) \\
3.05(\mathrm{t}, \mathrm{J}=6 \cdot 43)\end{array}$ & $0 \cdot 85-0.98(12 \mathrm{H}, \mathrm{c}), 1 \cdot 4-1 \cdot 7(3 \mathrm{H}, \mathrm{c}), 1 \cdot 70-1.93(1 \mathrm{H}, \mathrm{c})$ \\
\hline $\mathrm{i}-\operatorname{Pr}(\mathrm{M})$ & $\begin{array}{l}1.473(\mathrm{~s}) \\
1.487(\mathrm{~s})\end{array}$ & $3 \cdot 13-\cdot 24(\mathrm{c})$ & $\begin{array}{l}2 \cdot 73(\mathrm{~d}, \mathrm{~J}=6 \cdot 10) \\
2 \cdot 84(\mathrm{~d}, \mathrm{~J}=5 \cdot 45)\end{array}$ & $0.85-0.97(9 \mathrm{H}, \mathrm{c}), 1.23-1.40(1 \mathrm{H}, \mathrm{c}), 1.4-1.7(1 \mathrm{H}, \mathrm{c})$ \\
\hline $\mathrm{n}-\mathrm{Bu}(\mathrm{A})$ & $1 \cdot 489(\mathrm{~s})$ & $3 \cdot 23(\mathrm{t}, \mathrm{J}=7 \cdot 02)$ & $2 \cdot 98(\mathrm{t}, \mathrm{J}=6 \cdot 88)$ & $\begin{array}{l}0 \cdot 83-0 \cdot 97(9 \mathrm{H}, \text { undefined } \mathrm{m}), 1 \cdot 23-1 \cdot 38(4 \mathrm{H}, \mathrm{m}), 1 \cdot 4- \\
1 \cdot 7(5 \mathrm{H}, \mathrm{m})\end{array}$ \\
\hline $\mathrm{n}-\mathrm{Bu}(\mathrm{B})$ & $1 \cdot 475(\mathrm{~s})$ & $\begin{array}{c}3 \cdot 23,3 \cdot 26(\mathrm{dd}, \mathrm{J}=5 \cdot 61 \\
8 \cdot 09)\end{array}$ & $3 \cdot 04(t, J=6 \cdot 35)$ & $\begin{array}{l}0.89(3 \mathrm{H}, \mathrm{t}, \mathrm{J}=6.76), 0.92(6 \mathrm{H}, \mathrm{d}, \mathrm{J}=6.43), 1 \cdot 23- \\
1.38(4 \mathrm{H}, \mathrm{m}), 1.38-1 \cdot 70(5 \mathrm{H}, \mathrm{m})\end{array}$ \\
\hline $\mathrm{i}-\mathrm{Bu}(\mathrm{A})$ & $1 \cdot 492(\mathrm{~s})$ & $3 \cdot 23(\mathrm{t}, \mathrm{J}=7 \cdot 10)$ & $3 \cdot 04(\mathrm{t}, \mathrm{J}=7 \cdot 10)$ & $\begin{array}{l}0.83-0.98(12 \mathrm{H}, \text { undefined } \mathrm{m}), 1 \cdot 39(4 \mathrm{H}, \mathrm{t}, \mathrm{J}=6.93) \text {, } \\
1 \cdot 5-1 \cdot 7(2 \mathrm{H}, \mathrm{m})\end{array}$ \\
\hline $\mathrm{i}-\mathrm{Bu}(\mathrm{B})$ & $1 \cdot 476(\mathrm{~s})$ & $\begin{array}{c}3 \cdot 23,3: 26(\mathrm{dd}, \mathrm{J}=5 \cdot 62 \\
8 \cdot 09)\end{array}$ & $3.08(\mathrm{t}, \mathrm{J}=7 \cdot 10)$ & $0.92(12 \mathrm{H}, \mathrm{d}, \mathrm{J}=6 \cdot 27), 1 \cdot 35-1 \cdot 72(6 \mathrm{H}, \mathrm{m})$ \\
\hline sec-Bu (M) & $\begin{array}{l}1 \cdot 473(\mathrm{~s}) \\
1 \cdot 486(\mathrm{~s})\end{array}$ & $3 \cdot 11-3 \cdot 25(\mathrm{c})$ & $\begin{array}{l}2 \cdot 81(\mathrm{~d}, \mathrm{~J}=5 \cdot 93) \\
2 \cdot 86(\mathrm{~d}, \mathrm{~J}=4 \cdot 62) \\
2 \cdot 92(\mathrm{~d}, \mathrm{~J}=5 \cdot 12) \\
2 \cdot 99(\mathrm{~d}, \mathrm{~J}=4 \cdot 29)\end{array}$ & $\begin{array}{l}0 \cdot 82-0.97 \quad(12 \mathrm{H}, \quad \mathrm{c}), \quad 1 \cdot 08-1 \cdot 28 \quad(1 \mathrm{H}, \mathrm{c}), \quad 1 \cdot 3-1 \cdot 75 \\
(5 \mathrm{H}, \mathrm{c})\end{array}$ \\
\hline$\left(\mathrm{CH}_{2}\right)_{2-}$ & $\begin{array}{l}1.472(\mathrm{~s}) \\
1.486(\mathrm{~s})\end{array}$ & $3 \cdot 23(\mathrm{t}, \mathrm{J}=6 \cdot 93)$ & $\begin{array}{l}2 \cdot 98(\mathrm{t}, \mathrm{J}=6 \cdot 77) \\
3.04(\mathrm{t}, \mathrm{J}=6 \cdot 35)\end{array}$ & $\begin{array}{l}0.87-0.96(6 \mathrm{H}, \mathrm{c}), 1.4-1.7(3 \mathrm{H}, \mathrm{c}), 1 \cdot 7-1.9(2 \mathrm{H}, \mathrm{c}) \\
2 \cdot 12-2 \cdot 27(2 \mathrm{H}, \mathrm{c})\end{array}$ \\
\hline $\begin{array}{l}\mathrm{CO}_{2} \mathrm{Na}(\mathrm{M}) \\
\left(\mathrm{CH}_{2}\right)_{3-} \\
\mid \\
\mathrm{CO}_{2} \mathrm{Na}(\mathrm{M})\end{array}$ & $\begin{array}{l}1.473(\mathrm{~s}) \\
1.487(\mathrm{~s})\end{array}$ & $\begin{array}{c}\cdot 26(\mathrm{t}, \mathrm{J}=6 \cdot 68) \\
3 \cdot 23(\mathrm{t}, \mathrm{J}=6 \cdot 93) \\
3 \cdot 25(\mathrm{t}, \mathrm{t}-\mathrm{like})\end{array}$ & $2 \cdot 98,305$ (each t-like) & $\begin{array}{l}0 \cdot 86-0.96(6 \mathrm{H}, \mathrm{c}), 1 \cdot 4-1 \cdot 7(7 \mathrm{H}, \mathrm{c}), 2 \cdot 19(2 \mathrm{H}, \mathrm{t}, \mathrm{J}= \\
6 \cdot 59)\end{array}$ \\
\hline
\end{tabular}

Table 8. NMR spectra of substituted $N$-(carboxyalkyl)isoleucine tert-butyl ester.

\begin{tabular}{|c|c|c|c|c|}
\hline $\mathrm{R}$ & $\mathrm{Bu}^{\mathrm{t}}$ & $-\mathrm{CH}-$ & -NHCHR & Other protons \\
\hline $\mathrm{H}$ & $1 \cdot 49(\mathrm{~s})$ & $3 \cdot 17(\mathrm{~d}, \mathrm{~J}=5 \cdot 28)$ & $\begin{array}{c}3 \cdot 08,3 \cdot 15(\mathrm{dd}, \mathrm{J}=16 \cdot 5 \\
18 \cdot 3)\end{array}$ & $\begin{array}{l}0.92(3 \mathrm{H}, \mathrm{t}, \mathrm{J}=6 \cdot 68), 0.93(3 \mathrm{H}, \mathrm{d}, \mathrm{J}=6 \cdot 11), 1 \cdot 17-1 \cdot 57 \\
(2 \mathrm{H}, \mathrm{m})\end{array}$ \\
\hline $\operatorname{Me}(\mathrm{A})$ & $1.49(\mathrm{~s})$ & $3 \cdot 08(\mathrm{~d}, \mathrm{~J}=6 \cdot 1 \mathrm{l})$ & $3 \cdot 07(\mathrm{q}, \mathrm{J}=6 \cdot 92)$ & $\begin{array}{l}0.92(3 \mathrm{H}, \mathrm{t}, \mathrm{J}=7 \cdot 43), 0.92(3 \mathrm{H}, \mathrm{d} \mathrm{J}=7.09), 1 \cdot 22(3 \mathrm{H}, \\
\mathrm{d}, \mathrm{J}=6.92), 1.34-1.83(3 \mathrm{H}, \mathrm{m})\end{array}$ \\
\hline $\operatorname{Me}(\mathrm{B})$ & $1 \cdot 48(\mathrm{~s})$ & $3 \cdot 24(\mathrm{~d}, \mathrm{~J}=4 \cdot 95)$ & $3 \cdot 10(\mathrm{q}, \mathrm{J}=6 \cdot 93)$ & $\begin{array}{l}0.86-0.98(6 \mathrm{H}, \mathrm{c}), 1.23(3 \mathrm{H}, \mathrm{d}, \mathrm{J}=6.93), 1.38-1.83 \\
(3 \mathrm{H}, \mathrm{c})\end{array}$ \\
\hline Et $(\mathrm{A})$ & $1 \cdot 50(\mathrm{~s})$ & $3.03(\mathrm{~d}, \mathrm{~J}=6.44)$ & $\begin{array}{c}2 \cdot 88,2 \cdot 91(\mathrm{dd}, \mathrm{J}=5 \cdot 69 \\
7 \cdot 67)\end{array}$ & $\begin{array}{l}0.89(3 \mathrm{H}, \mathrm{t}, \mathrm{J}=7.43), 0.90(3 \mathrm{H}, \mathrm{t}, \mathrm{J}=7.01), 0.91(3 \mathrm{H}, \\
\mathrm{d}, \mathrm{J}=6.92), 1 \cdot 16-1.80(5 \mathrm{H}, \mathrm{c})\end{array}$ \\
\hline Et $(B)$ & $1 \cdot 48(\mathrm{~s})$ & $3 \cdot 16(\mathrm{~d}, \mathrm{~J}=5 \cdot 28)$ & $2 \cdot 95(\mathrm{~d}, \mathrm{~J}=6 \cdot 27)$ & $\begin{array}{l}0.89(3 \mathrm{H}, \mathrm{t}, \mathrm{J}=7 \cdot 26), 0.92(3 \mathrm{H}, \mathrm{t}, \mathrm{J}=7 \cdot 26), 0.92(3 \mathrm{H}, \\
\mathrm{d}, \mathrm{J}=6.60), 1 \cdot 19-1 \cdot 65(5 \mathrm{H}, \mathrm{c})\end{array}$ \\
\hline $\mathrm{n}-\operatorname{Pr}(\mathrm{A})$ & $1 \cdot 50(\mathrm{~s})$ & $3.01(\mathrm{~d}, \mathrm{~J}=6 \cdot 60)$ & $\begin{array}{c}2 \cdot 95,2 \cdot 97(\mathrm{dd}, \mathrm{J}=6 \cdot 19 \\
7 \cdot 18)\end{array}$ & $\begin{array}{l}0.90(3 \mathrm{H}, \mathrm{t}, \mathrm{J}=6 \cdot 68), 0.91(3 \mathrm{H}, \mathrm{t}, \mathrm{J}=7 \cdot 18), 0.91(3 \mathrm{H}, \\
\mathrm{d}, \mathrm{J}=6 \cdot 26), 1 \cdot 71-1 \cdot 82(7 \mathrm{H}, \mathrm{c})\end{array}$ \\
\hline $\mathrm{n}-\operatorname{Pr}(\mathrm{B})$ & $1 \cdot 48(\mathrm{~s})$ & $3 \cdot 16(\mathrm{~d}, \mathrm{~J}=5 \cdot 11)$ & $2.99(\mathrm{t}, \mathrm{J}=6.36)$ & $\begin{array}{l}0.91(3 \mathrm{H}, \mathrm{t}, \mathrm{J}=7.26), 0.91(3 \mathrm{H}, \mathrm{d}, \mathrm{J}=6.59), 0.92(3 \mathrm{H}, \\
\mathrm{t}, \mathrm{J}=6.82), 1.18-1.80(7 \mathrm{H}, \mathrm{c})\end{array}$ \\
\hline i- $\operatorname{Pr}(\mathrm{A})$ & $1 \cdot 50(\mathrm{~s})$ & $2 \cdot 94(\mathrm{~d}, \mathrm{~J}=7 \cdot 26)$ & $2 \cdot 69(\mathrm{~d}, \mathrm{~J}=6 \cdot 27)$ & $\begin{array}{l}0.90(3 \mathrm{H}, \mathrm{t}, \mathrm{J}=7.34), 0.90,0.91(6 \mathrm{H}, \text { each } \mathrm{d}, \mathrm{J}=6.92) \text {, } \\
1.26-1.88(4 \mathrm{H}, \mathrm{c})\end{array}$ \\
\hline $\mathrm{i}-\operatorname{Pr}(\mathrm{B})$ & $1 \cdot 48(\mathrm{~s})$ & $3 \cdot 12(\mathrm{~d}, \mathrm{~J}=5 \cdot 11)$ & $2 \cdot 76(d, J=5 \cdot 77)$ & $\begin{array}{l}0.92(12 \mathrm{H}, \mathrm{t}-\mathrm{like}, \mathrm{J}=6 \cdot 85), 1 \cdot 20-1.35(1 \mathrm{H}, \mathrm{m}), 1 \cdot 65 \\
1.93(3 \mathrm{H}, \mathrm{m})\end{array}$ \\
\hline $\mathrm{n}-\mathrm{Bu}(\mathrm{A})$ & $1 \cdot 50(\mathrm{~s})$ & $3 \cdot 01(\mathrm{~d}, \mathrm{~J}=6 \cdot 44)$ & $\begin{array}{c}2 \cdot 93,2 \cdot 96(\mathrm{dd}, \mathrm{J}=6 \cdot 02 \\
7 \cdot 51)\end{array}$ & $\begin{array}{l}0.88(3 \mathrm{H}, \mathrm{t}, \mathrm{J}=6 \cdot 85), 0 \cdot 90(3 \mathrm{H}, \mathrm{t}, \mathrm{J}=7 \cdot 26), 0.91(3 \mathrm{H}, \\
\mathrm{d}, \mathrm{J}=6 \cdot 42), 1 \cdot 20-1 \cdot 80(9 \mathrm{H}, \mathrm{c})\end{array}$ \\
\hline $\mathrm{n}-\mathrm{Bu}(\mathrm{B})$ & $1 \cdot 48(\mathrm{~s})$ & $3 \cdot 16(\mathrm{~d}, \mathrm{~J}=5 \cdot 11)$ & $2.99(\mathrm{t}, \mathrm{J}=6.35)$ & $\begin{array}{l}0.89(3 \mathrm{H}, \mathrm{t}, \mathrm{J}=6.76), 0.91(3 \mathrm{H}, \mathrm{d}, \mathrm{J}=6.76),(0.92(3 \mathrm{H}, \\
\mathrm{t}, \mathrm{J}=7 \cdot 26), 1 \cdot 20-1 \cdot 80(9 \mathrm{H}, \mathrm{c})\end{array}$ \\
\hline $\mathrm{i}-\mathrm{Bu}(\mathrm{A})$ & $1 \cdot 50(\mathrm{~s})$ & $3 \cdot 00(\mathrm{~d}, \mathrm{~J}=6 \cdot 60)$ & $3 \cdot 00(\mathrm{t}, \mathrm{J}=7 \cdot 18)$ & $\begin{array}{l}0.85-0.95(12 \mathrm{H}, \mathrm{m}), 1 \cdot 15-1 \cdot 35(1 \mathrm{H}, \mathrm{m}), 1 \cdot 40(2 \mathrm{H}, \mathrm{t} \text {-like, } \\
\mathrm{J}=6 \cdot 77), 1 \cdot 45-1 \cdot 85(3 \mathrm{H}, \mathrm{m})\end{array}$ \\
\hline $\mathrm{i}-\mathrm{Bu}(\mathrm{B})$ & $1 \cdot 48(\mathrm{~s})$ & $3 \cdot 16(\mathrm{~d}, \mathrm{~J}=5 \cdot 11)$ & $3.03(\mathrm{t}, \mathrm{J}=7 \cdot 01)$ & $\begin{array}{l}0.89-0.95(12 \mathrm{H}, \mathrm{m}), 1 \cdot 21-1 \cdot 37(1 \mathrm{H}, \mathrm{m}), 1 \cdot 42(2 \mathrm{H}, \mathrm{t}, \mathrm{J}= \\
6.93), 1 \cdot 40-1 \cdot 75(3 \mathrm{H}, \mathrm{m})\end{array}$ \\
\hline sec-Bu (A) & $1 \cdot 50(\mathrm{~s})$ & $2 \cdot 96(\mathrm{~d}, \mathrm{~J}=7 \cdot 26)$ & $\begin{array}{l}2 \cdot 80(\mathrm{~d}, \mathrm{~J}=5.94) \\
2 \cdot 82(\mathrm{~d}, \mathrm{~J}=5 \cdot 11)\end{array}$ & $\begin{array}{l}0.85-0.95(9 \mathrm{H}, \mathrm{m}), 1 \cdot 08-1 \cdot 34(2 \mathrm{H}, \mathrm{m}), 1 \cdot 36-1 \cdot 68(4 \mathrm{H}, \\
\mathrm{m})\end{array}$ \\
\hline sec-Bu (B) & $1 \cdot 47(\mathrm{~s})$ & $\begin{array}{l}3 \cdot 09(\mathrm{~d}, \mathrm{~J}=5 \cdot 28) \\
3 \cdot 11(\mathrm{~d}, \mathrm{~J}=5 \cdot 11)\end{array}$ & $\begin{array}{l}2 \cdot 85(\mathrm{~d}, \mathrm{~J}=5 \cdot 44) \\
2 \cdot 92(\mathrm{~d}, \mathrm{~J}=4 \cdot 46)\end{array}$ & $\begin{array}{l}0 \cdot 84-0.96(9 \mathrm{H}, \mathrm{m}), 1 \cdot 10-1 \cdot 36(2 \mathrm{H}, \mathrm{m}), 1 \cdot 36-1 \cdot 80(4 \mathrm{H} \text {, } \\
\mathrm{m})\end{array}$ \\
\hline
\end{tabular}


Table 8 (continued).

\begin{tabular}{|c|c|c|c|c|}
\hline $\mathrm{R}$ & $\mathrm{Bu}^{\mathrm{t}}$ & $-\mathrm{CH}-$ & -NHCHR & Other protons \\
\hline$\left(\mathrm{CH}_{2}\right)_{2-}$ & $1.50(\mathrm{~s})$ & $3 \cdot 03(\mathrm{~d}, \mathrm{~J}=6 \cdot 43)$ & $\begin{array}{c}2 \cdot 94,2 \cdot 96(\mathrm{dd}, \mathrm{J} \\
7 \cdot 43)\end{array}$ & $\begin{array}{l}0.90(3 \mathrm{H}, \mathrm{t}, \mathrm{J}=6 \cdot 92), 0.91(3 \mathrm{H}, \mathrm{d}, \mathrm{J}=7 \cdot 26), 1 \cdot 15-1 \cdot 35 \\
(1 \mathrm{H}, \mathrm{m}), 1 \cdot 70-1 \cdot 90(4 \mathrm{H}, \mathrm{m}), 2 \cdot 10-2 \cdot 25(2 \mathrm{H}, \mathrm{m})\end{array}$ \\
\hline $\begin{array}{l}\mathrm{CO}_{2} \mathrm{Na}(\mathrm{A}) \\
\left(\mathrm{CH}_{2}\right)_{2-} \\
\mid\end{array}$ & $1 \cdot 48(\mathrm{~s})$ & $3 \cdot 17(\mathrm{~d}, \mathrm{~J}=4 \cdot 95)$ & $2.99(\mathrm{t}, \mathrm{J}=6 \cdot 44)$ & $\begin{array}{l}0.92(3 \mathrm{H}, \mathrm{t}, \mathrm{J}=7 \cdot 26), 0.92(3 \mathrm{H}, \mathrm{d}, \mathrm{J}=6 \cdot 93), 1 \cdot 15-1 \cdot 30 \\
(1 \mathrm{H}, \mathrm{m}), 1 \cdot 40-1 \cdot 65(5 \mathrm{H}, \mathrm{m}), 2 \cdot 15-2 \cdot 30(2 \mathrm{H}, \mathrm{m})\end{array}$ \\
\hline $\begin{array}{l}\mathrm{CO}_{2} \mathrm{Na}(\mathrm{B}) \\
\left(\mathrm{CH}_{2}\right)_{3-}- \\
\mid\end{array}$ & $1 \cdot 50(\mathrm{~s})$ & $3 \cdot 02(\mathrm{~d}, \mathrm{~J}=6 \cdot 43)$ & $2 \cdot 95(\mathrm{t}, \mathrm{J}=6 \cdot 10)$ & $\begin{array}{l}0.90(3 \mathrm{H}, \mathrm{t}, \mathrm{J}=7 \cdot 26), 0 \cdot 91(3 \mathrm{H}, \mathrm{d}, \mathrm{J}=6 \cdot 60), 1 \cdot 15-1 \cdot 30 \\
(1 \mathrm{H}, \mathrm{m}), 1 \cdot 40-1 \cdot 65(5 \mathrm{H}, \mathrm{m}), 1 \cdot 65-1 \cdot 80(1 \mathrm{H}, \mathrm{m}), 2 \cdot 19\end{array}$ \\
\hline $\begin{array}{l}\mathrm{CO}_{2} \mathrm{Na}(\mathrm{A}) \\
\left(\mathrm{CH}_{2}\right)_{3}-\end{array}$ & $1 \cdot 48(\mathrm{~s})$ & $3 \cdot 17(\mathrm{~d}, \mathrm{~J}=4.95)$ & $3.00($ undefined $t)$ & $\begin{array}{l}(2 \mathrm{H}, \mathrm{t}, \mathrm{J}=7 \cdot 09) \\
0 \cdot 91(3 \mathrm{H}, \mathrm{d}, \mathrm{J}=6 \cdot 76), 0 \cdot 92(3 \mathrm{H}, \mathrm{t}, \mathrm{J}=7 \cdot 26), 1 \cdot 15-1 \cdot 45 \\
(1 \mathrm{H}, \mathrm{m}), 1 \cdot 40-1 \cdot 80(6 \mathrm{H}, \mathrm{m}), 2 \cdot 19(2 \mathrm{H}, \text { undefined } \mathrm{t})\end{array}$ \\
\hline $\mathrm{CO}_{2} \mathrm{Na}(\mathrm{B})$ & & & & \\
\hline
\end{tabular}

Table 9. NMR spectra of substituted $N$-(carboxyalkyl) serine-O-tert-butyl ester.

\begin{tabular}{|c|c|c|c|c|}
\hline $\mathrm{R}$ & $\mathrm{Bu}^{\mathrm{t}}$ & $-\mathrm{CH}-$ & -NHCHR & Other protons \\
\hline $\mathrm{H}$ & $1 \cdot 490(\mathrm{~s})$ & $3 \cdot 38(t, J=6 \cdot 02)$ & $\begin{array}{c}3 \cdot 14,3 \cdot 22(2 \mathrm{H}, \mathrm{dd}, \mathrm{J}= \\
16 \cdot 5,20 \cdot 8)\end{array}$ & $1 \cdot 21(9 \mathrm{H}, \mathrm{s}), 3 \cdot 61,3 \cdot 63(2 \mathrm{H}$, each $\mathrm{d}, \mathrm{J}=6 \cdot 02)$ \\
\hline $\mathrm{Me}(\mathrm{M})$ & $\begin{array}{l}1.487(\mathrm{~s}) \\
1.491(\mathrm{~s})\end{array}$ & $3 \cdot 38(\mathrm{t}, \mathrm{J}=6 \cdot 60)$ & $\begin{array}{l}3 \cdot 16(\mathrm{q}, \mathrm{J}=6 \cdot 93) \\
3 \cdot 19(\mathrm{q}, \mathrm{J}=7 \cdot 09)\end{array}$ & $\begin{array}{l}1 \cdot 206,1 \cdot 214(9 \mathrm{H}, \text { each s}), 1 \cdot 23(3 \mathrm{H}, \mathrm{d}, \mathrm{J}=7 \cdot 00), 3 \cdot 52- \\
3.69(2 \mathrm{H}, \mathrm{c})\end{array}$ \\
\hline Et $(\mathbf{M})$ & $\begin{array}{l}1 \cdot 486(\mathrm{~s}) \\
1 \cdot 494(\mathrm{~s})\end{array}$ & $3 \cdot 34(\mathrm{t}, \mathrm{J}=6 \cdot 60)$ & $\begin{array}{l}3 \cdot 01,3 \cdot 03(\mathrm{dd}, \mathrm{J}=5 \cdot 86 \\
7 \cdot 34), 3 \cdot 08(\mathrm{t}, . \mathrm{J}=6 \cdot 36)\end{array}$ & $\begin{array}{l}0.88,0.89(3 \mathrm{H}, \text { each } \mathrm{t}, \mathrm{J}=7.51), 1 \cdot 20-1 \cdot 21(9 \mathrm{H}, \text { each } \mathrm{s}) \\
1.45-1.75(2 \mathrm{H}, \mathrm{c}), 3.52-3.69(2 \mathrm{H}, \mathrm{c})\end{array}$ \\
\hline $\mathrm{n}-\operatorname{Pr}(\mathrm{M})$ & $\begin{array}{l}1.485(\mathrm{~s}) 3 \\
1.495(\mathrm{~s})\end{array}$ & $\begin{array}{c}33,3 \cdot 35(\mathrm{dd}, \mathrm{J}=5 \cdot 20 \\
6 \cdot 85)\end{array}$ & $\begin{array}{l}3 \cdot 08(\mathrm{t}, \mathrm{J}=6 \cdot 60) \\
3 \cdot 12(\mathrm{t}, \mathrm{J}=6 \cdot 35)\end{array}$ & $\begin{array}{l}0.91(3 \mathrm{H}, \mathrm{t}, \mathrm{J}=7 \cdot 26), 1 \cdot 2-1 \cdot 6(4 \mathrm{H}, \mathrm{c}), 1 \cdot 206,1 \cdot 213(9 \mathrm{H}, \\
\text { each s), } 3 \cdot 51-3 \cdot 54(2 \mathrm{H}, \mathrm{c})\end{array}$ \\
\hline i- $\operatorname{Pr}(\mathrm{M})$ & $\begin{array}{l}1 \cdot 484(\mathrm{~s}) \\
1 \cdot 495(\mathrm{~s}) 3\end{array}$ & $\begin{array}{c}3 \cdot 28(t, \mathrm{~J}=6 \cdot 60) \\
28,3 \cdot 31(\mathrm{dd}, \mathrm{J}=4 \cdot 95 \\
10 \cdot 06)\end{array}$ & $\begin{array}{l}2 \cdot 82(\mathrm{~d}, \mathrm{~J}=6 \cdot 10) \\
2 \cdot 90(\mathrm{~d}, \mathrm{~J}=5 \cdot 60)\end{array}$ & $\begin{array}{l}0.92,0.95(3 \mathrm{H}, \text { each } \mathrm{d}, \mathrm{J}=6.93,6 \cdot 76), 1 \cdot 207,1 \cdot 213(9 \mathrm{H}, \\
\text { each s }), 1.85(1 \mathrm{H}, \text { sep, } \mathrm{J}=6.76), 3 \cdot 53-3.68(2 \mathrm{H}, \mathrm{c})\end{array}$ \\
\hline $\mathrm{n}-\mathrm{Bu}(\mathrm{A})$ & $1 \cdot 495(\mathrm{~s})$ & $3 \cdot 33(\mathrm{t}, \mathrm{J}=6 \cdot 68)$ & $3 \cdot 06(\mathrm{t}, \mathrm{J}=6 \cdot 60)$ & $\begin{array}{l}0.88(3 \mathrm{H}, \mathrm{t}, \mathrm{J}=6 \cdot 77), 1 \cdot 21(9 \mathrm{H}, \mathrm{s}), 1 \cdot 23-1 \cdot 36(4 \mathrm{H}, \mathrm{m}), \\
1 \cdot 47-1 \cdot 65(2 \mathrm{H}, \mathrm{m}), 3 \cdot 52-3 \cdot 65(2 \mathrm{H}, \mathrm{m})\end{array}$ \\
\hline $\mathrm{n}-\mathrm{Bu}(\mathrm{B})$ & $1 \cdot 486(\mathrm{~s}) 3$ & $\begin{array}{c}33,3.36(\mathrm{dd}, \mathrm{J}=5 \cdot 44 \\
6 \cdot 76)\end{array}$ & $3 \cdot 12(\mathrm{t}, \mathrm{J}=6 \cdot 52)$ & $\begin{array}{l}0.89(3 \mathrm{H}, \mathrm{t}, \mathrm{J}=6.68), 1.21(9 \mathrm{H}, \mathrm{s}), 1 \cdot 2-1 \cdot 4(4 \mathrm{H}, \mathrm{m}), \\
1.5-1.64(2 \mathrm{H}, \mathrm{m}), 3 \cdot 52-3.68(2 \mathrm{H}, \mathrm{m})\end{array}$ \\
\hline $\mathrm{i}-\mathrm{Bu}(\mathrm{A})$ & $1 \cdot 498(\mathrm{~s})$ & $3 \cdot 32(\mathrm{t}, \mathrm{J}=6 \cdot 68)$ & $3 \cdot 11(\mathrm{t}, \mathrm{J}=7 \cdot 09)$ & $\begin{array}{l}0 \cdot 91,0 \cdot 92(6 \mathrm{H}, \text { each d, } \mathrm{J}=6 \cdot 43,6 \cdot 60), 1 \cdot 21(9 \mathrm{H}, \mathrm{s}), 1 \cdot 41 \\
(2 \mathrm{H}, \mathrm{t}-\mathrm{like}, \mathrm{J}=6 \cdot 77), 1 \cdot 64-1 \cdot 70(1 \mathrm{H}, \mathrm{m}), 3 \cdot 53-3 \cdot 65 \\
(2 \mathrm{H}, \mathrm{m})\end{array}$ \\
\hline $\mathrm{i}-\mathrm{Bu}(\mathrm{B})$ & $1 \cdot 488(\mathrm{~s}) 3$ & 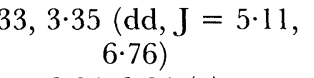 & $3 \cdot 15(t, J=7 \cdot 01)$ & $\begin{array}{l}0.92(6 \mathrm{H}, \mathrm{d}, \mathrm{J}=6.60), 1.21(9 \mathrm{H}, \mathrm{s}), 1.34-1.53(2 \mathrm{H}, \mathrm{m}), \\
1.53-1.68(1 \mathrm{H}, \mathrm{m}), 3.52-3.69(2 \mathrm{H}, \mathrm{m})\end{array}$ \\
\hline sec-Bu $(M)$ & $\begin{array}{l}1 \cdot 485(\mathrm{~s}) \\
1 \cdot 495(\mathrm{~s})\end{array}$ & $3 \cdot 24-3 \cdot 34(\mathrm{c})$ & $2 \cdot 89-3 \cdot 08(c)$ & $\begin{array}{l}0.82-0.97(6 \mathrm{H}, \mathrm{c}), 1 \cdot 207,1 \cdot 214(9 \mathrm{H}, \text { each s}), 1 \cdot 40-1.75 \\
(3 \mathrm{H}, \mathrm{c}), 2 \cdot 89-3.08(1 \mathrm{H}, \mathrm{c}), 3 \cdot 53-3.68(2 \mathrm{H}, \mathrm{c})\end{array}$ \\
\hline$\left(\mathrm{CH}_{2}\right)_{2}-$ & $\begin{array}{l}1 \cdot 184(\mathrm{~s}) \\
1 \cdot 495(\mathrm{~s})\end{array}$ & $3 \cdot 3-3 \cdot 4(c)$ & $\begin{array}{l}3 \cdot 07(\mathrm{t}, \mathrm{J}=6 \cdot 68) \\
3 \cdot 10(\mathrm{t}, \mathrm{J}=6 \cdot 52)\end{array}$ & $\begin{array}{l}1 \cdot 207,1 \cdot 213(9 \mathrm{H}, \text { each s}), 1 \cdot 73-1 \cdot 89(2 \mathrm{H}, \mathrm{c}), 2 \cdot 13-2 \cdot 27 \\
(2 \mathrm{H}, \mathrm{c}), 3 \cdot 52-3 \cdot 70(2 \mathrm{H}, \mathrm{c})\end{array}$ \\
\hline $\begin{array}{l}\mathrm{CO}_{2} \mathrm{Na}(\mathrm{M}) \\
\left(\mathrm{CH}_{2}\right)_{3-} \\
\mid \\
\mathrm{CO}_{2} \mathrm{Na}(\mathrm{M})\end{array}$ & $\begin{array}{l}1 \cdot 484(\mathrm{~s}) \\
1 \cdot 495(\mathrm{~s})\end{array}$ & $3 \cdot 29-3 \cdot 38(\mathrm{c})$ & $3 \cdot 01-3 \cdot 15(c)$ & $\begin{array}{l}1 \cdot 205,1 \cdot 212(9 \mathrm{H}, \text { each s}), \text { ca. } 1 \cdot 51(4 \mathrm{H}, \text { br. s }), \text { ca. } 2 \cdot 20 \\
(2 \mathrm{H}, \text { br. t }), 3 \cdot 52-3 \cdot 69(2 \mathrm{H}, \mathrm{c})\end{array}$ \\
\hline
\end{tabular}

Table 10. NMR spectra of substituted $N$-(carboxyalkyl)threonine-O-tert-butyl ester.

\begin{tabular}{|c|c|c|c|c|}
\hline $\mathrm{R}$ & $\mathrm{Bu}^{\mathrm{t}}$ & $-\mathrm{CH}-$ & -NHCHR & Other protons \\
\hline $\mathrm{H}$ & $1 \cdot 50(\mathrm{~s})$ & $3 \cdot 21(\mathrm{~d}, \mathrm{~J}=6 \cdot 93)$ & $\begin{array}{c}3 \cdot 05,3 \cdot 17(2 \mathrm{H}, \mathrm{dd}, \mathrm{J}= \\
16 \cdot 4,32 \cdot 4)\end{array}$ & $\begin{array}{l}1 \cdot 19(3 \mathrm{H}, \mathrm{d}, \mathrm{J}=6 \cdot 42), 1 \cdot 27(9 \mathrm{H}, \mathrm{s}), 3 \cdot 96(1 \mathrm{H}, \text { quin, } \mathrm{J}= \\
6 \cdot 44)\end{array}$ \\
\hline $\operatorname{Me}(\mathrm{M})$ & $\begin{array}{l}1 \cdot 492(\mathrm{~s}) \\
1 \cdot 499(\mathrm{~s})\end{array}$ & $\begin{array}{l}3 \cdot 11(\mathrm{~d}, \mathrm{~J}=7 \cdot 58) \\
3 \cdot 34(\mathrm{~d}, \mathrm{~J}=5 \cdot 77)\end{array}$ & $3 \cdot 00-3 \cdot 14(\mathrm{c})$ & $\begin{array}{l}1 \cdot 12-1 \cdot 30(6 \mathrm{H}, \mathrm{c}), 1 \cdot 25,1 \cdot 27(9 \mathrm{H}, \text { each s}), 3 \cdot 83-4 \cdot 03 \\
(1 \mathrm{H}, \mathrm{c})\end{array}$ \\
\hline Et $(\mathrm{A})$ & $1.50(\mathrm{~s})$ & $3.08(\mathrm{~d}, \mathrm{~J}=7 \cdot 42)$ & $\begin{array}{c}2 \cdot 89,2 \cdot 92(\mathrm{dd}, \mathrm{J}=5 \cdot 28 \\
7 \cdot 92)\end{array}$ & $\begin{array}{l}0.88,(3 \mathrm{H}, \mathrm{t}, \mathrm{J}=7.51), 1 \cdot 17(3 \mathrm{H}, \mathrm{d}, \mathrm{J}=6.27), 1.27(9 \mathrm{H}, \\
\mathrm{s}), 1.40-1.75(2 \mathrm{H}, \mathrm{m}), 3 \cdot 87-3.92(1 \mathrm{H}, \mathrm{m})\end{array}$ \\
\hline Et $(B)$ & $1 \cdot 49(\mathrm{~s})$ & $3 \cdot 29(\mathrm{~d}, \mathrm{~J}=5 \cdot 94)$ & $3 \cdot 00(\mathrm{t}, \mathrm{J}=6 \cdot 03)$ & $\begin{array}{l}0.87(3 \mathrm{H}, \mathrm{t}, \mathrm{J}=7.51), 1 \cdot 19(3 \mathrm{H}, \mathrm{d}, \mathrm{J}=6.44), 1 \cdot 26(9 \mathrm{H}, \\
\mathrm{s}), 1.54-1.74(2 \mathrm{H}, \mathrm{m}), 3.98(1 \mathrm{H}, \text { quin, } \mathrm{J}=6 \cdot 27)\end{array}$ \\
\hline $\mathrm{n}-\operatorname{Pr}(\mathrm{M})$ & $\begin{array}{l}1 \cdot 49(\mathrm{~s}) \\
1 \cdot 50(\mathrm{~s})\end{array}$ & $\begin{array}{l}3.07(\mathrm{~d}, \mathrm{~J}=7 \cdot 59) \\
3.29(\mathrm{~d}, \mathrm{~J}=5 \cdot 94)\end{array}$ & $\begin{array}{l}2 \cdot 94,2 \cdot 97(\mathrm{dd}, \mathrm{J}=5 \cdot 77 \\
7 \cdot 42), 3 \cdot 03(\mathrm{t}, \mathrm{J}=6 \cdot 60)\end{array}$ & $\begin{array}{l}0 \cdot 88,0 \cdot 93(3 \mathrm{H}, \text { each } \mathrm{t}, \mathrm{J}=7 \cdot 26), 1 \cdot 16,1 \cdot 19(6 \mathrm{H}, \text { each } \mathrm{d}, \\
\mathrm{J}=4 \cdot 76,4 \cdot 95), 1 \cdot 26,1 \cdot 27(9 \mathrm{H}, \text { each } \mathrm{s}), 1 \cdot 30-1 \cdot 70(4 \mathrm{H}, \\
\text { c), } 3 \cdot 89,3 \cdot 97(1 \mathrm{H}, \text { each quin, } \mathrm{J}=6 \cdot 3)\end{array}$ \\
\hline
\end{tabular}


Table 10 (continued).

\begin{tabular}{|c|c|c|c|c|}
\hline $\mathrm{R}$ & $\mathrm{Bu}^{\mathrm{t}}$ & $-\mathrm{CH}-$ & -NHCHR & Other protons \\
\hline \multirow[t]{2}{*}{$\mathrm{i}-\operatorname{Pr}(\mathrm{M})$} & $1 \cdot 49(\mathrm{~s})$ & $3.05(\mathrm{~d}, \mathrm{~J}=7.09)$ & $2 \cdot 71(\mathrm{~d}, \mathrm{~J}=6 \cdot 11)$ & \multirow{2}{*}{$\begin{array}{l}0.90(3 \mathrm{H}, \mathrm{d}, \mathrm{J}=6 \cdot 93), 0 \cdot 96(3 \mathrm{H}, \mathrm{d}, \mathrm{J}=6 \cdot 60), 1 \cdot 17,1 \cdot 19 \\
(3 \mathrm{H}, \text { each } \mathrm{d}, \mathrm{J}=6 \cdot 10), 1.25,1 \cdot 27(9 \mathrm{H}, \text { each } \mathrm{s}), 1 \cdot 7-2 \cdot 0 \\
(1 \mathrm{H}, \mathrm{c}), 3 \cdot 92,4 \cdot 00(1 \mathrm{H}, \text { each quin })\end{array}$} \\
\hline & $1 \cdot 50(\mathrm{~s})$ & $3 \cdot 26(\mathrm{~d}, \mathrm{~J}=5 \cdot 78)$ & $2 \cdot 80(\mathrm{~d}, \mathrm{~J}=5 \cdot 61)$ & \\
\hline \multirow[t]{2}{*}{$\mathrm{n}-\mathrm{Bu}(\mathrm{M})$} & $1.49(\mathrm{~s})$ & $3.07(\mathrm{~d}, \mathrm{~J}=7 \cdot 42)$ & $3.02(t, J=6 \cdot 19), 2 \cdot 93$ & \multirow{4}{*}{$\begin{array}{l}0 \cdot 83-0.93(6 \mathrm{H}, \mathrm{c}), 1 \cdot 26,1 \cdot 27(9 \mathrm{H} \text {, each } \mathrm{s}), 1 \cdot 14-1 \cdot 65 \\
(6 \mathrm{H}, \mathrm{c}), 3 \cdot 89,3.97(1 \mathrm{H}, \text { each quin, } \mathrm{J}=6 \cdot 85,6 \cdot 35) \\
0 \cdot 85-0.97(9 \mathrm{H}, \mathrm{c}), 1 \cdot 25,1 \cdot 26(9 \mathrm{H}, \text { each } \mathrm{s}), 1 \cdot 12-1 \cdot 25 \\
(2 \mathrm{H}, \mathrm{c}), 3 \cdot 89,3.96(1 \mathrm{H}, \text { each quin, } \mathrm{J}=6 \cdot 76,6 \cdot 27)\end{array}$} \\
\hline & $1.50(\mathrm{~s})$ & $3.29(\mathrm{~d}, \mathrm{~J}=6 \cdot 11)$ & $2.96(\mathrm{dd}, \mathrm{J}=5 \cdot 77,7 \cdot 59)$ & \\
\hline \multirow[t]{2}{*}{$\mathrm{i}-\mathrm{Bu}(\mathrm{M})$} & $1 \cdot 49(\mathrm{~s})$ & $3.06(\mathrm{~d}, \mathrm{~J}=7 \cdot 42)$ & $2 \cdot 93-3.06(\mathrm{c})$ & \\
\hline & $1 \cdot 51(\mathrm{~s})$ & $3.30(\mathrm{~d}, \mathrm{~J}=5.94)$ & & \\
\hline \multirow[t]{4}{*}{ sec-Bu $(\mathrm{M})$} & $1.48(\mathrm{~s})$ & $3.03(\mathrm{~d}, \mathrm{~J}=8.08)$ & $2 \cdot 80(\mathrm{~d}, \mathrm{~J}=5 \cdot 77)$ & \multirow{4}{*}{$\begin{array}{l}0.82-0.97(6 \mathrm{H}, \mathrm{c}), 1 \cdot 1-1 \cdot 3(2 \mathrm{H}, \mathrm{c}), 1 \cdot 25,1 \cdot 26,1 \cdot 27(9 \mathrm{H}, \\
\text { each s), } 1.32-1.74(1 \mathrm{H}, \mathrm{c}), 3 \cdot 84-4.05(1 \mathrm{H}, \mathrm{c})\end{array}$} \\
\hline & $1.49(\mathrm{~s})$ & $3.06(\mathrm{~d}, \mathrm{~J}=7 \cdot 43)$ & $2.83(\mathrm{~d}, \mathrm{~J}=4.95)$ & \\
\hline & $1 \cdot 50(\mathrm{~s})$ & $3 \cdot 24(\mathrm{~d}, \mathrm{~J}=5 \cdot 77)$ & $2 \cdot 90(\mathrm{~d}, \mathrm{~J}=5 \cdot 27)$ & \\
\hline & & $3.25(\mathrm{~d}, \mathrm{~J}=5.94)$ & $2.95(\mathrm{~d}, \mathrm{~J}=4.45)$ & \\
\hline \multirow{2}{*}{$\left(\mathrm{CH}_{2}\right)_{2-}$} & $1.49(\mathrm{~s})$ & $3.09(\mathrm{~d}, \mathrm{~J}=7 \cdot 26)$ & $2 \cdot 94,2 \cdot 96(\mathrm{dd}, \mathrm{J}=5 \cdot 69$ & \multirow{6}{*}{$\begin{array}{l}1 \cdot 17,1 \cdot 19(3 \mathrm{H}, \text { each } \mathrm{d}, \mathrm{J}=6 \cdot 43,6 \cdot 44), 1 \cdot 25,1 \cdot 27(9 \mathrm{H}, \\
\text { each s }), 1 \cdot 70-1 \cdot 94(2 \mathrm{H}, \mathrm{c}), 2 \cdot 18(2 \mathrm{H}, \mathrm{t}, \mathrm{J}=7 \cdot 92), 3 \cdot 91 \text {, } \\
4 \cdot 00(1 \mathrm{H}, \text { quin, } 6 \cdot 68,6 \cdot 11) \\
1 \cdot 17,1 \cdot 19(3 \mathrm{H}, \text { each d, J }=6 \cdot 10,5 \cdot 78), 1 \cdot 25,1 \cdot 27(9 \mathrm{H}, \\
\text { each s), } 1 \cdot 4-1 \cdot 7(4 \mathrm{H}, \mathrm{c}), 2 \cdot 12-2 \cdot 24(2 \mathrm{H}, \text { undefined } \mathrm{t}) \\
3 \cdot 89,3 \cdot 98(1 \mathrm{H}, \text { each quin, } \mathrm{J}=6 \cdot 85,6 \cdot 19)\end{array}$} \\
\hline & $1 \cdot 50(\mathrm{~s})$ & $3.29(\mathrm{~d}, \mathrm{~J}=5 \cdot 61)$ & $7 \cdot 67)$ & \\
\hline \multirow{4}{*}{$\begin{array}{l}\mathrm{CO}_{2} \mathrm{Na}(\mathrm{M}) \\
\left(\mathrm{CH}_{2}\right)_{3-} \\
\mathrm{CO}_{2} \mathrm{Na}(\mathrm{M})\end{array}$} & & & & \\
\hline & $1.49(\mathrm{~s})$ & $3 \cdot 07(\mathrm{~d}, \mathrm{~J}=7 \cdot 42)$ & $2 \cdot 94(\mathrm{t}, \mathrm{J}=6 \cdot 02)$ & \\
\hline & $1.50(\mathrm{~s})$ & $3.29(\mathrm{~d}, \mathrm{~J}=5 \cdot 94)$ & $3.03(\mathrm{t}, \mathrm{J}=5 \cdot 53)$ & \\
\hline & & & & \\
\hline
\end{tabular}

Table 11. NMR spectra of substituted $N$-(carboxyalkyl)aspartic acid tert-butyl esters.

\begin{tabular}{|c|c|c|c|c|}
\hline $\mathrm{R}$ & $\mathrm{Bu}^{\mathrm{t}}$ & $-\mathrm{CH}-$ & -NHCHR & Other protons \\
\hline $\mathrm{H}$ & $\begin{array}{l}1 \cdot 481(\mathrm{~s}) \\
1.492(\mathrm{~s})\end{array}$ & $\begin{array}{l}3 \cdot 53(\mathrm{~d}, \mathrm{~J}=5 \cdot 12) \\
356(\mathrm{~d}, \mathrm{~J}=5 \cdot 11)\end{array}$ & $3 \cdot 19,3 \cdot 20(2 \mathrm{H}$, each $\mathrm{s})$ & $2 \cdot 6-2 \cdot 8(2 \mathrm{H}, \mathrm{m})$ \\
\hline $\operatorname{Me}(\mathrm{M})$ & $1.459(\mathrm{~s}), 1.470(\mathrm{~s})$ & $3 \cdot 53(\mathrm{t}, \mathrm{J}=5 \cdot 12)$ & $3 \cdot 20(\mathrm{q}, \mathrm{J}=6 \cdot 98)$ & $1 \cdot 22,1.23(3 \mathrm{H}$, each $\mathrm{d}, \mathrm{J}=6.92), 2 \cdot 56$ \\
\hline Et $(\mathrm{M})$ & $\begin{array}{l}1.4 / 3(\mathrm{~s}), 1.482(\mathrm{~s}) \\
1.461(\mathrm{~s}), 1.468(\mathrm{~s}) \\
1.474(2), 1.484(\mathrm{~s})\end{array}$ & $\begin{array}{c}3.56(t, J=5.11) \\
3.46-3.57(\mathrm{c})\end{array}$ & $\begin{array}{l}3 \cdot 23(\mathrm{q}, \mathrm{J}=6 \cdot \mathrm{y3}) \\
3 \cdot 06(\mathrm{t}, \mathrm{J}=6 \cdot 27) \\
3 \cdot 10(\mathrm{t}, \mathrm{J}=6 \cdot 43)\end{array}$ & $\begin{array}{l}2 \cdot 80(2 \mathrm{H}, \mathrm{c}) \\
0.89(3 \mathrm{H}, \mathrm{t}, \mathrm{J}=7.51), 1.5-1.7(2 \mathrm{H}, \mathrm{c}), \\
2 \cdot 6-2 \cdot 8(2 \mathrm{H}, \mathrm{c})\end{array}$ \\
\hline $\mathrm{n}-\operatorname{Pr}(\mathrm{M})$ & $\begin{array}{l}1 \cdot 460(\mathrm{~s}) \\
1 \cdot 468(\mathrm{~s}) \\
1 \cdot 474(\mathrm{~s}) \\
1.485(\mathrm{~s})\end{array}$ & $\begin{array}{l}3 \cdot 47,3 \cdot 49 \\
(\mathrm{dd}, \mathrm{J}=6 \cdot 19,7 \cdot 51) \\
3 \cdot 52,3 \cdot 55 \\
(\mathrm{dd}, \mathrm{J}=5 \cdot 03,7 \cdot 84)\end{array}$ & $\begin{array}{l}3 \cdot 11(t, J=6 \cdot 44) \\
3 \cdot 16(t, J=6 \cdot 52)\end{array}$ & $\begin{array}{l}0.91(3 \mathrm{H}, \mathrm{t}, \mathrm{J}=7 \cdot 26), 1 \cdot 2-1 \cdot 6(4 \mathrm{H}, \mathrm{c}), \\
2 \cdot 6-2 \cdot 8(2 \mathrm{H}, \mathrm{c})\end{array}$ \\
\hline $\mathrm{i}-\operatorname{Pr}(\mathrm{M})$ & $\begin{array}{l}1.462(\mathrm{~s}) \\
1.466(\mathrm{~s}) \\
1.476(\mathrm{~s}) \\
1.486(\mathrm{~s})\end{array}$ & $\begin{array}{l}3 \cdot 41,3 \cdot 43 \\
(\mathrm{dd}, \mathrm{J}=6 \cdot 11,7 \cdot 26) \\
3 \cdot 48,3 \cdot 51 \\
(\mathrm{dd}, \mathrm{J}=4 \cdot 86,7 \cdot 67)\end{array}$ & $\begin{array}{l}2 \cdot 87(\mathrm{~d}, \mathrm{~J}=5 \cdot 61) \\
2 \cdot 93(\mathrm{~d}, \mathrm{~J}=5 \cdot 94)\end{array}$ & $\begin{array}{l}0.88-0.96(6 \mathrm{H}, \mathrm{c}), 1.78-1.93(1 \mathrm{H}, \mathrm{c}), \\
2 \cdot 63-2.78(2 \mathrm{H}, \mathrm{c})\end{array}$ \\
\hline $\mathrm{n}-\mathrm{Bu}(\mathrm{M})$ & $\begin{array}{l}1.461(\mathrm{~s}) \\
1.469(\mathrm{~s}) \\
1.475(\mathrm{~s}) \\
1.486(\mathrm{~s})\end{array}$ & $\begin{array}{l}3 \cdot 47,3 \cdot 49 \\
(\mathrm{dd}, \mathrm{J}=6 \cdot 02,7 \cdot 34) \\
3 \cdot 52,3 \cdot 55 \\
(\mathrm{dd}, \mathrm{J}=5 \cdot 11,7 \cdot 59)\end{array}$ & $\begin{array}{l}3 \cdot 10(t, J=6 \cdot 35) \\
3 \cdot 14(t, J=6 \cdot 52)\end{array}$ & $0 \cdot 89(3 \mathrm{H}, \mathrm{t}, \mathrm{J}=6 \cdot 85), 1 \cdot 2-2 \cdot 8(8 \mathrm{H}, \mathrm{c})$ \\
\hline $\mathrm{i}-\mathrm{Bu}(\mathrm{M})$ & $\begin{array}{l}1.459(\mathrm{~s}) \\
1.467(\mathrm{~s}) \\
1.476(\mathrm{~s}) \\
1.487(\mathrm{~s})\end{array}$ & $\begin{array}{l}3 \cdot 48,3 \cdot 51 \\
(\mathrm{dd}, \mathrm{J}=6 \cdot 02,7 \cdot 34) \\
3 \cdot 50,3 \cdot 58 \\
(\mathrm{dd}, \mathrm{J}=4 \cdot 95,7 \cdot 59)\end{array}$ & $\begin{array}{l}3 \cdot 13(\mathrm{t}, \mathrm{J}=7 \cdot 09) \\
3 \cdot 20(\mathrm{t}, \mathrm{J}=7 \cdot 01)\end{array}$ & $\begin{array}{l}0.92,0 \cdot 93(6 \mathrm{H}, \text { each d, J }=6 \cdot 43), 1 \cdot 55- \\
1 \cdot 75(1 \mathrm{H}, \mathrm{c}), 1 \cdot 3-1 \cdot 5(2 \mathrm{H}, \mathrm{c}), 2 \cdot 6-2 \cdot 8 \\
(2 \mathrm{H}, \mathrm{c})\end{array}$ \\
\hline sec-Bu $(\mathrm{M})$ & $\begin{array}{l}1.461(\mathrm{~s}), 1.466(\mathrm{~s}) \\
1.476(\mathrm{~s}), 1.484(\mathrm{~s}) \\
1.486(\mathrm{~s})\end{array}$ & $3.35-3.55(\mathrm{c})$ & $2 \cdot 9-3 \cdot 1(\mathrm{c})$ & $\begin{array}{l}0 \cdot 80-0 \cdot 95(6 \mathrm{H}, \mathrm{c}), 1 \cdot 1-1 \cdot 3(1 \mathrm{H}, \mathrm{c}), 1 \cdot 5- \\
1 \cdot 7(2 \mathrm{H}, \mathrm{c}), 2 \cdot 60-2 \cdot 75(2 \mathrm{H}, \mathrm{c})\end{array}$ \\
\hline$\left(\mathrm{CH}_{2}\right)_{2}-$ & $\begin{array}{l}1.460(\mathrm{~s}), 1.464(\mathrm{~s}) \\
1.469(\mathrm{~s}), 1.472(\mathrm{~s})\end{array}$ & $3 \cdot 45-3 \cdot 60(\mathrm{c})$ & $3 \cdot 05-3 \cdot 20(\mathrm{c})$ & $\begin{array}{l}1 \cdot 7-1 \cdot 85(2 \mathrm{H}, \mathrm{c}), 2 \cdot 15-2 \cdot 25(2 \mathrm{H}, \mathrm{c}), 2 \cdot 65- \\
2 \cdot 75(2 \mathrm{H}, \mathrm{c})\end{array}$ \\
\hline $\begin{array}{l}\mathrm{CO}_{2} \mathrm{Na}(\mathrm{M}) \\
\left(\mathrm{CH}_{2}\right)_{3-}\end{array}$ & $\begin{array}{l}1.485(\mathrm{~s}) \\
1.459(\mathrm{~s}), 1.466(\mathrm{~s}) \\
1.472(\mathrm{~s}), 1.485(\mathrm{~s})\end{array}$ & $3 \cdot 45-3 \cdot 60(\mathrm{c})$ & $3 \cdot 15$ (br. s) & $1 \cdot 55(4 \mathrm{H}, \mathrm{c}), 2.20(2 \mathrm{H}, \mathrm{c}), 2 \cdot 6-2 \cdot 8(2 \mathrm{H}, \mathrm{c})$ \\
\hline $\mathrm{CO}_{2} \mathrm{Na}(\mathrm{M})$ & & & & \\
\hline
\end{tabular}

$\left([\alpha]_{\mathrm{D}}\right)$ and CD spectra were measured with JASCO DP1 181 and J-20 (Japan Spectroscopic Co., Ltd) spectrophotomers, respectively. ${ }^{1} \mathrm{H}$ NMR spectra were measured with a JEOL JNM-GX270 spectrometer using 3-(trimethylsilyl)propionic acid $\mathrm{d}_{4}$-sodium salt as an internal standard. Personal computers were NEG PC-9801 and Fujitsu FM 77AV. The instrument used for HPLG analysis was a Shimadzu LC-5A, equipped with a
Nucleosil 5C18 column $(4 \times 250 \mathrm{~mm})$ and operated as follows: flow rate $0.7 \mathrm{ml} / \mathrm{min}$; detector $\mathrm{UV}-235 \mathrm{~nm}$; chart speed $2.5 \mathrm{~mm} / \mathrm{min}$; mobile phase, a mixture of $0.05 \mathrm{M}$ aqAcONa and $\mathrm{MeOH}(3: 7, \mathrm{v} / \mathrm{v})$. N-(Carboxy-3-methyl)butyl-L-leucine disodium salt (7).

Both isomers (6-a and 6-b) of N-(3-methyl-l-sodioxycarbonyl)-butyl-L-leucine tert-butyl ester, which were 
Table 12. Circular dichroism (CD) and [alpha] $]_{D}$ of substituted $N$-(carboxyalkyl)amino acid tert-butyl esters.

\begin{tabular}{|c|c|c|c|c|}
\hline \multicolumn{2}{|c|}{ Substituents* } & \multirow{2}{*}{$\begin{array}{l}\text { Retention } \\
\text { time† (min) }\end{array}$} & \multirow{2}{*}{$\begin{array}{l}{[\alpha]_{\mathrm{D}}\left(24^{\circ}\right)} \\
\left(\mathrm{c} \text { in } \mathrm{H}_{2} \mathrm{O}\right)\end{array}$} & \multirow{2}{*}{$\begin{array}{c}\mathrm{CD} \\
\lambda_{\max } \mathrm{nm}\left(\Delta \varepsilon \text { in } \mathrm{H}_{2} \mathrm{O}\right)\end{array}$} \\
\hline $\mathrm{R}_{1}$ & $\mathrm{R}_{\mathrm{s}}$ & & & \\
\hline \multirow[t]{5}{*}{$\mathrm{i}-\mathrm{Pr}$} & $\mathrm{H}$ & $3 \cdot 81$ & $-13 \cdot 4(0 \cdot 62)$ & $210(+0.92)$ \\
\hline & $\mathrm{n}-\mathrm{Bu}(\mathrm{A})$ & $5 \cdot 23$ & $-23 \cdot 3(0 \cdot 68)$ & $229(-0 \cdot 56)$ \\
\hline & $\mathrm{n}-\mathrm{Bu}(\mathrm{B})$ & $7 \cdot 25$ & $-14.9(0.65)$ & $223(+0.67)$ \\
\hline & $\mathrm{i}-\mathrm{Bu}(\mathrm{A})$ & $5 \cdot 20$ & $-27.8(0.49)$ & $229(-0.56)$ \\
\hline & $\mathrm{i}-\mathrm{Bu}(\mathrm{B})$ & $7 \cdot 05$ & $-12 \cdot 5(0.44)$ & $223(+0 \cdot 76)$ \\
\hline \multirow[t]{5}{*}{$\mathrm{i}-\mathrm{Bu}$} & $\mathrm{H}$ & $4 \cdot 34$ & $-5 \cdot 7(0.68)$ & $210(+0 \cdot 81)$ \\
\hline & $\mathrm{n}-\mathrm{Bu}(\mathrm{A})$ & $6 \cdot 09$ & $-15 \cdot 1(0 \cdot 64)$ & $234(-0 \cdot 16)$ \\
\hline & $\mathrm{n}-\mathrm{Bu}(\mathrm{B})$ & $8 \cdot 91$ & $-6.6(0.56)$ & $221(+0.58)$ \\
\hline & $\mathrm{i}-\mathrm{Bu}(\mathrm{A})$ & $6 \cdot 05$ & $-19 \cdot 3(0.62)$ & $237(-0 \cdot 11)$ \\
\hline & $\mathrm{i}-\mathrm{Bu}(\mathrm{B})$ & $8 \cdot 99$ & $-3.9(0.47)$ & $223(+0 \cdot 63)$ \\
\hline \multirow[t]{9}{*}{ sec-Bu } & $\mathrm{H}$ & $4 \cdot 36$ & $-3 \cdot 8(0 \cdot 63)$ & $211(+1 \cdot 16)$ \\
\hline & $\operatorname{Me}(\mathrm{A})$ & $4 \cdot 33$ & $-24 \cdot 8(0.56)$ & $230(-0 \cdot 19)$ \\
\hline & $\mathrm{Me}(\mathrm{B})$ & $4 \cdot 79$ & $-15.7(0.37)$ & $224(+1.52), 271(-0.97)$ \\
\hline & Et $(\mathrm{A})$ & $4 \cdot 60$ & $-26 \cdot 3(1.03)$ & $228(-0.37)$ \\
\hline & Et (B) & $5 \cdot 70$ & $+0.5(1.08)$ & $220(+1 \cdot 15)$ \\
\hline & $\mathrm{n}-\mathrm{Bu}(\mathrm{A})$ & $6 \cdot 29$ & $-19 \cdot 9(1 \cdot 14)$ & $231(-0 \cdot 35)$ \\
\hline & $\mathrm{n}-\mathrm{Bu}(\mathrm{B})$ & $8 \cdot 94$ & $-6.2(0.95)$ & $224(+1 \cdot 00)$ \\
\hline & $\mathrm{i}-\mathrm{Bu}(\mathrm{A})$ & $6 \cdot 36$ & $-24.6(0.89)$ & $231(-0.35)$ \\
\hline & $\mathrm{i}-\mathrm{Bu}(\mathrm{B})$ & $8 \cdot 73$ & $-3.9(1.00)$ & $223(+0.93)$ \\
\hline \multirow{2}{*}{$\mathrm{CH}-\mathrm{CH}_{3}$} & $\mathrm{H}$ & $4 \cdot 59$ & $-9.6(0.53)$ & $233(-0 \cdot 13)$ \\
\hline & Et $(\mathrm{A})$ & $4 \cdot 83$ & $-25 \cdot 0(0.60)$ & $227(-0.83)$ \\
\hline $\mathrm{OBu}^{\mathrm{t}}$ & Et (B) & $6 \cdot 03$ & $+8.0(0.67)$ & $222(+0.45)$ \\
\hline
\end{tabular}

(A) and (B) denote the 1st and 2nd eluted products by an Amberlite XAD-2 column.

* $\mathrm{R}_{1}-\mathrm{CH}-\mathrm{CO}_{2} \mathrm{Bu}^{\mathrm{t}} \quad \dagger \mathrm{HPLC}$ analytical conditions are described in the experimental part.<smiles>[R]C(NC)C(=O)O[Na]</smiles>

obtained by the automated synthesis described in the text, were further purified on a XAD-2 column using $50 \%$ methanol as an eluent. The eluted solutions were evaporated to half of their volume under reduced pressure, and then freeze-dried. To the purified powder of 6 -a $(0.3 \mathrm{~g})$ was added chilled trifluoroacetic acid $(5 \mathrm{ml})$ and the mixture was stirred at room temperature for 2 hours. After evaporation of trifluoroacetic acid under reduced pressure, the residue was dissolved in water $(5$ $\mathrm{ml}$ ) and treated with $1 \mathrm{~N}$ aq- $\mathrm{NaOH}$ to adjust it to $\mathrm{pH} 11$. The resulting solution was charged onto a column of Sephadex LH-20 $(35 \times 500 \mathrm{~mm})$ and eluted with water to obtain N-(1-carboxy-3-methyl)butyl-L-leucine disodium salt $(0.21 \mathrm{~g} ; 7$-a $)$ after lyophilization. Found: C, $44 \cdot 37 ; \mathrm{H}$, $7 \cdot 27 ; \mathrm{N}, 4 \cdot 43 \%$. Calcd for $\mathrm{C}_{12} \mathrm{H}_{21} \mathrm{NNa}_{2} \mathrm{O}_{4} \cdot 2 \mathrm{H}_{2} \mathrm{O}: \mathrm{C}$, $44.31 ; \mathrm{H}, 7 \cdot 75 ; \mathrm{N}, 4 \cdot 31 \% .{ }^{1} \mathrm{H}$ NMR (ppm in $\left.\mathrm{D}_{2} \mathrm{O}\right)[\delta]:$ $0.916(6 \mathrm{H}, \mathrm{d}, \mathrm{j}=6.43), 0.935(6 \mathrm{H}, \mathrm{d}, \mathrm{J}=6.43), 1.540$ $(4 \mathrm{H}, \mathrm{t}, \mathrm{J}=6.93), 1.672(2 \mathrm{H}$, sextet, $\mathrm{J}=6.63), 3.289(2 \mathrm{H}$, $\mathrm{t}, \mathrm{J}=7 \cdot 09) .[\alpha]_{\mathrm{D}} 0^{\circ}\left(\mathrm{c}=0.43\right.$ in $\left.\mathrm{H}_{2} \mathrm{O}, 20^{\circ} \mathrm{C}\right)$. IR $[v] \mathrm{KBr}$ $\mathrm{cm}^{-1}: 2960,1619,1446,1409$ and 1383. In a similar manner, 7-b isomer $(0 \cdot 19 \mathrm{~g})$ of $\mathrm{N}$-(1-carboxy-3-mehtyl)butyl-L-leucine disodium salt was obtained starting from 6-b $(0 \cdot 3 \mathrm{~g})$. Found: C, 43.88; H, 7.45; N, 4.08\%. Calcd for $\mathrm{C}_{12} \mathrm{H}_{21} \mathrm{NNa}_{2} \mathrm{O}_{4} .2 \mathrm{H}_{2} \mathrm{O}: \mathrm{C}, 44 \cdot 31 ; \mathrm{H}, 7 \cdot 75 ; \mathrm{N}, 4 \cdot 31 \% .{ }^{1} \mathrm{H}$ NMR (ppm in $\left.\mathrm{D}_{2} \mathrm{O}\right)$ [ $\left.\mathrm{v}\right]: 0.938(6 \mathrm{H}, \mathrm{d}, \mathrm{J}=6.44), 0.947$ $(6 \mathrm{H}, \mathrm{D}, \mathrm{J}=6 \cdot 27), 1 \cdot 50-1 \cdot 78(6 \mathrm{H}$, complex m), $3 \cdot 392$
$(2 \mathrm{H}, \mathrm{t}, \mathrm{J}=6 \cdot 85) \cdot[\alpha]_{\mathrm{D}}+39 \cdot 2^{\circ}\left(\mathrm{c}=0 \cdot 47\right.$ in $\left.\mathrm{H}_{2} \mathrm{O}, 20^{\circ}\right)$. IR $[v]^{\mathrm{KBr}} \mathrm{cm}^{-1}: 2958,1629,1452$ and 1411 .

\section{References}

1. Hayashi, N., Sugawara, T., Shintani, M. and Katao, S., Journal Automatic Chemistry, 11 (1989), 212.

2. Hayashi, N., Farumashia, 26 (1900), 931.

3. Hayashi, N. and Sugawara, T., Chemical Letters (1988), 1613.

4. Hayashi, N. and Sugawara, T., Tetra. Computer Methods, 1 (1989), 237.

5. Winicov, H., Schainbaum, J., Bugkley, J., Longino, G., Hill, J. and Berkoff, C. E., Chimica Acta, 103 (1978), 469.

6. Legrand, M. and Bolla, P., Journal of Automatic Chemistry, 7 (1985), 31.

7. Chodosh, D. F., Woziegkowski, F. E., Shainbaum, J. and Berkoff, G. E., Journal of Automatic Chemistry, 5 (1983), 99.

8. Frisbee, A. R., Nantz, M. H., Kramer, G. W. and Fuchs, P. L., Journal of American Chemical Society., 106 (1984), 7143.

9. Ohfune, Y and Kurokawa, N., Journal of Synthetic Organic Chemistry (Japan), 44 (1986), 647.

10. Sangster, A. W., Thomas, S. E. and Tingling, N. L., Tetrahedron, 31 (1978), 878.

11. Kasi, T. and Sakamura, S., Agricultural Biological Chemistry, 45 (1981), 1483.

12. Mryazawa, T., Bulletin of the Chemical Society (Japan), 53 (1980), 2555 and 3661. 


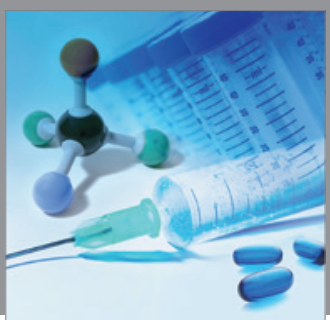

International Journal of

Medicinal Chemistry

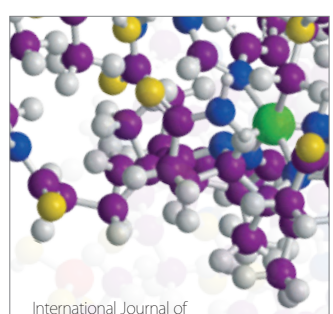

Carbohydrate Chemistry

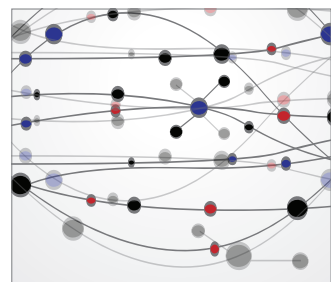

The Scientific World Journal
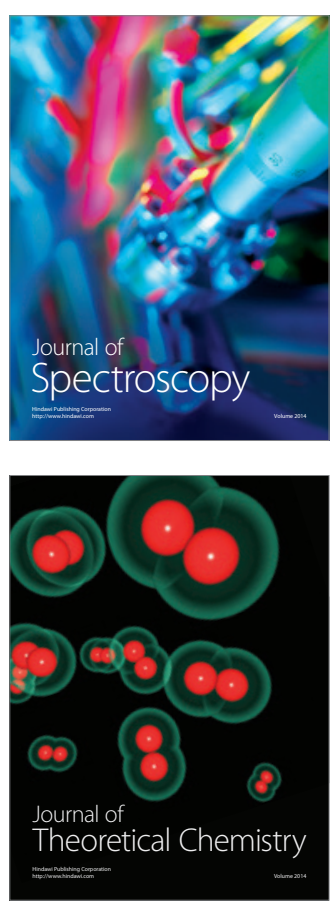
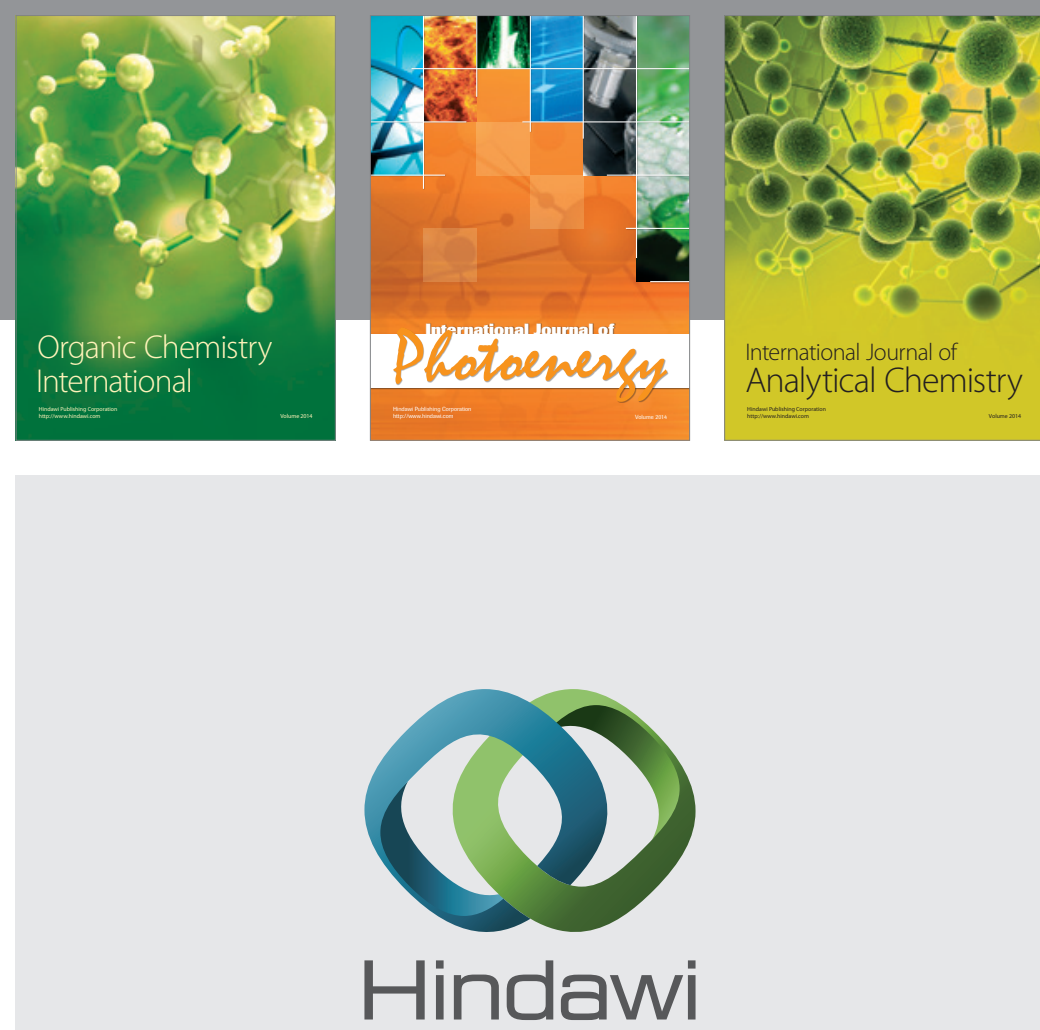

Submit your manuscripts at

http://www.hindawi.com
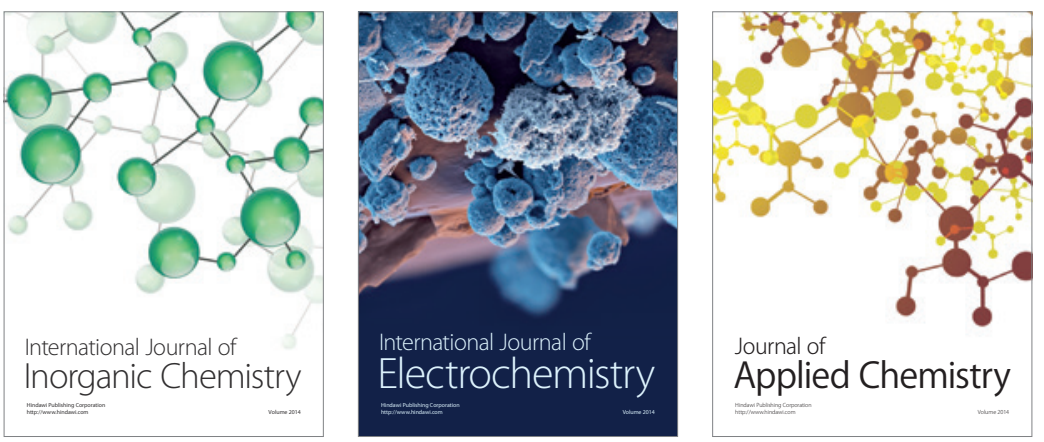

Journal of

Applied Chemistry
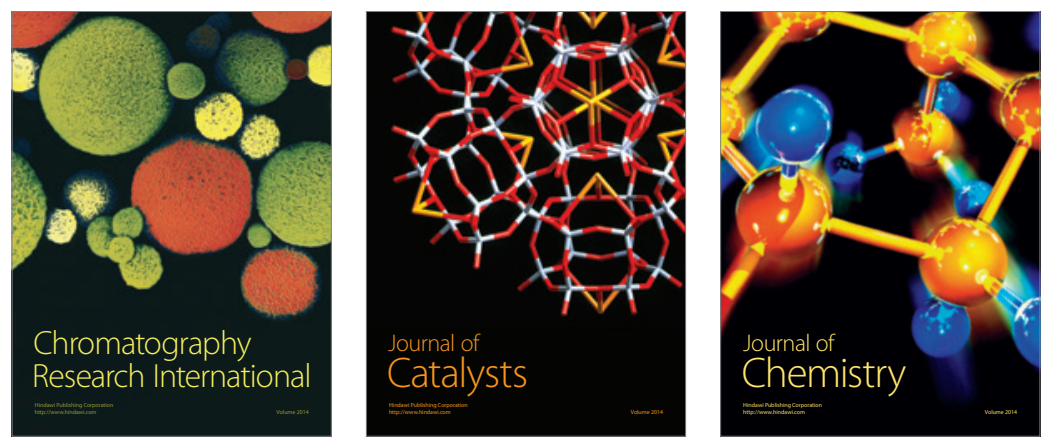
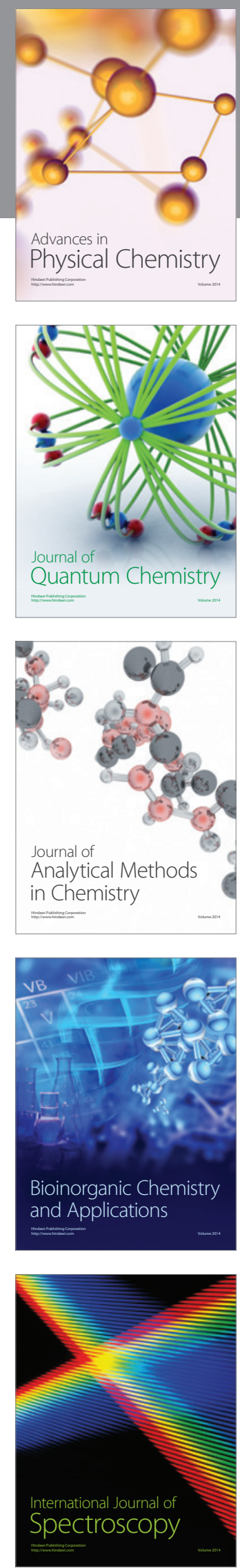